\title{
A checklist of agarics (gilled mushrooms) of Kerala State, India
}

\section{Adnaan Farook V, Shabeer Khan S and Manimohan $\mathbf{P}^{*}$}

\author{
Department of Botany, University of Calicut, Kerala, 673 635, India
}

Farook VA, Khan SS, Manimohan P 2013 - A checklist of agarics (gilled mushrooms) of Kerala State, India. Mycosphere 4(1), 97-131, Doi 10.5943 /mycosphere/4/1/6

\begin{abstract}
A literature-based checklist of agarics (gilled mushrooms) occurring in Kerala State, India is provided. It consists of 616 species of gilled mushrooms belonging in 112 genera and 5 orders (Agaricales, Boletales, Gomphales, Polyporales, Russulales) of the Class Agaricomycetes, Phylum Basidiomycota. The relevant original information is contained in about 122 papers, many with only limited distribution and availability. Most of these papers have been published during the last two decades. The geographical distribution of the reports covers all 14 districts of the State. At the order level, Agaricales has the highest number of species (579), followed by Russulales (21). The six most represented genera are Hygrocybe, Entoloma (41 spp. each), Lepiota (39 spp.), Marasmius (27 spp.), Leucocoprinus and Pluteus (22 spp. each). Several of the species are known only from the region and there is a possibility that many are endemic to the region as the level of endemism in the flora and fauna of Kerala is very high. This list covers only a part of the actual diversity of gilled mushrooms in Kerala as studies on the agarics of this region have been uneven, inadequate and therefore inconclusive.
\end{abstract}

Key words - Agaricomycetes - Basidiomycota - biodiversity - species

\author{
Article Information \\ Received 15 December 2012 \\ Accepted 4 January 2013 \\ Published online 10 February 2013 \\ *Corresponding author: P Manimohan - e-mail - pmanimohan@gmail.com
}

\section{Introduction}

Kerala State is a narrow strip of land on the south-west corner of the Indian Peninsula. It lies between north latitudes $8^{\circ} 18^{\prime}$ and $12^{\circ} 48^{\prime}$ and east longitudes $74^{\circ} 52^{\prime}$ and $72^{\circ} 22^{\prime}$ and is wedged between the Arabian Sea of the Indian Ocean and a mountain range called the Western Ghats. Most of the forested areas of the State are parts of the Western Ghats, which is one of the biodiversity hotspots of the world that has been very recently added to the World Heritage list. The State's total area is 38,863 sq $\mathrm{km}$, which is only about $1.8 \%$ of the total geographical area of India. Yet, almost a quarter of India's plant species are found in Kerala. Among the 4,667 vascular plant species of the State (Sasidharan 2004), 344 are endemic (Reddy et al. 2007). The very high biological diversity of Kerala is primarily due to the highly diversified ecological niches that occur from sea level up to more than 2,600 meters above sea level encompassing evergreen forests (wet evergreen and semievergreen climax forests, west coast tropical evergreen forests, southern hill top tropical evergreen forests and semi-evergreen forests), deciduous forests (secondary dry deciduous forests, southern dry deciduous forests, secondary moist deciduous forests and dry deciduous forests), shola forests, grasslands, mangroves, and the subtypes such as dry teak forests, lateritic semi-evergreen forests, littoral forests, myristica swamps, Nilgiri subtropical hill forests, south Indian subtropical hill 
Mycosphere Doi 10.5943/mycosphere/4/1/6

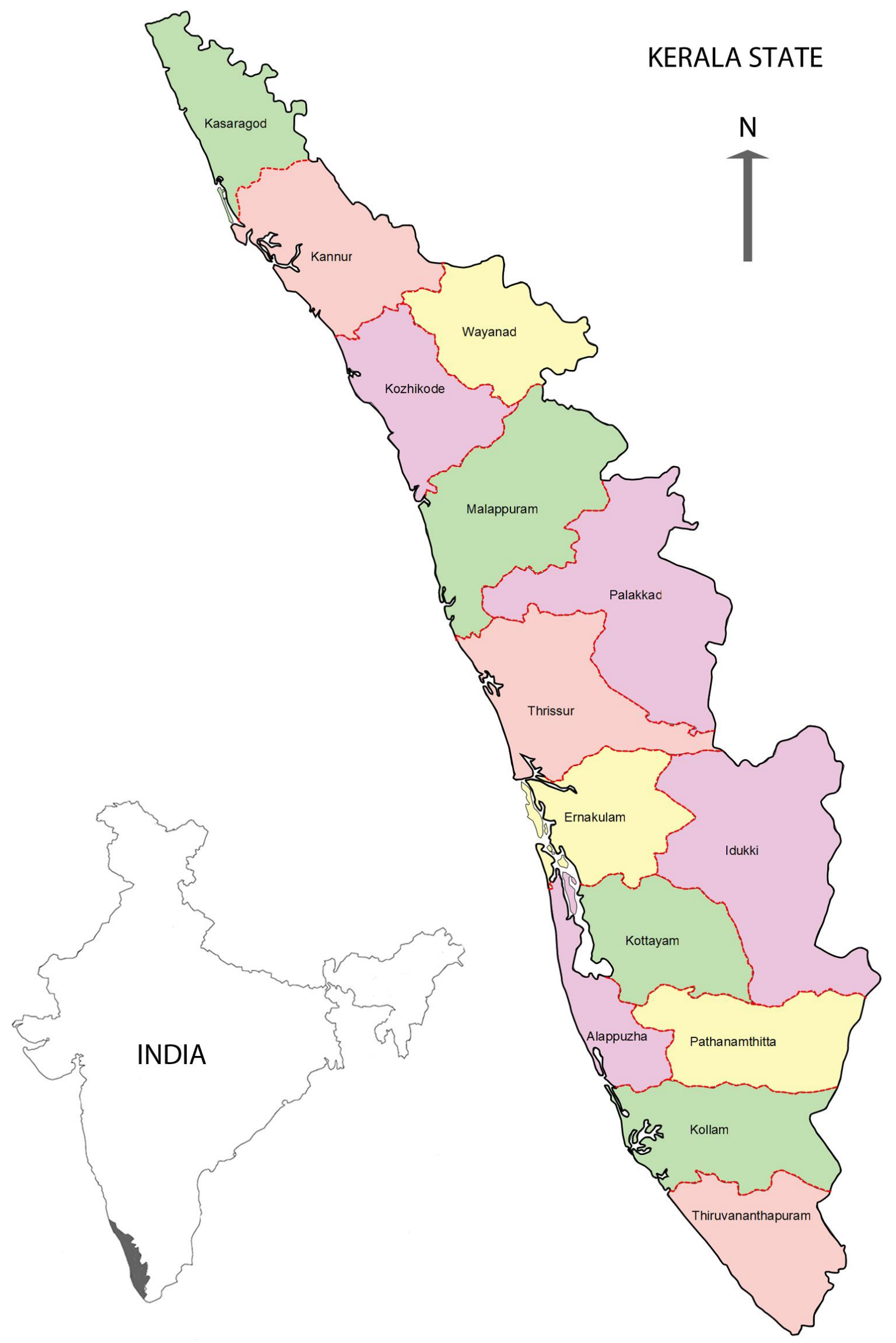

Fig. 1 - Map of Kerala State, India. 
savannah, southern moist mixed deciduous forests, southern secondary moist mixed deciduous forests, and very moist teak forests. The climate of Kerala is tropical, maritime and monsoonal.

It is obvious from the above account that Kerala must be harbouring a luxuriant mycota. However, to this day, the region has not been explored for many groups of fungi. Diversity of only very few groups of fungi such as soil microfungi, foliicolous microfungi, polypores, marine fungi, and lichens have been studied at least at a preliminary level. There are several reasons for this scenario, the scarcity of trained mycologists in the State being a major one. One group of fungi that has been rather intensively explored in the past three decades in this region by several workers is the agarics or gilled mushrooms. In order to consolidate the information so far made available on the diversity of agarics of this region and to contribute to the knowledge of mycota of Kerala, here we provide a literature-based checklist of agarics occurring in Kerala State. Gilled mushrooms are a morphologically discernable and not a taxonomically coherent group and hence they belong to several orders such as Agaricales, Boletales, Gomphales, Polyporales and Russulales of the Class Agaricomycetes of the Phylum Basidiomycota.

\section{Methods}

This checklist contains information gathered solely from published sources up to 31 December 2011. We have excluded all material presented only in Masters, MPhil and $\mathrm{PhD}$ dissertations and not subsequently published. Also, we have not listed species identified in the original sources only to genus level (e.g. Cortinarius sp.). No herbaria data are given in this checklist as that information is available in the references indicated. However, it must be noted that several mycological herbaria in Europe (e.g. Royal Botanic Gardens, Kew-K, Nationaal Herbarium Nederland, Leiden-L) and North America (e.g. Field Museum of Natural History, Chicago-F, New York Botanical Garden-NY) contain a significant number of agaric collections from Kerala. The systematic arrangement and the nomenclature used are those of Index
Fungorum (www.indexfungorum.org) as accessed on $25^{\text {th }}$ November 2012. In this checklist, the names of some species as reported in the cited publications have been replaced by currently accepted name according to the Species Fungorum website (www.speciesfungorum.org) and this has been indicated in such cases. Invalidly proposed new species from the region are excluded from this list. The checklist is organized alphabetically by order, family, genus and species.

\section{Results and Discussions}

Table 1 lists 616 species of gilled mushrooms belonging in 112 genera and 5 orders (Agaricales, Boletales, Gomphales, Polyporales and Russulales) of the Class Agaricomycetes of the Phylum Basidiomycota reported as occurring in Kerala State on the basis of published information. The relevant original information is contained in about 122 papers, many with only limited distribution and availability. Most of these papers have been published during the last two decades. The geographical distribution of the reports covers all the 14 districts of the State (see Fig 1.) At the order level, Agaricales has the highest number of species (579), followed by Russulales (21). The six most represented genera are Hygrocybe, Entoloma (41 spp. each), Lepiota (39 spp.), Marasmius (27 spp.), Leucocoprinus and Pluteus (22 spp. each). Several of the listed species (138) were described as new to science and so far many of them are known only from Kerala. There is a possibility that many are endemic to the region as the level of endemism in the flora and fauna of Kerala is very high.

Although the list displayed in Table 1 might appear extensive, it covers only a part of the actual diversity of gilled mushrooms in Kerala. For example, although only six species of the genus Mycena are listed in this checklist, a work in progress in our lab has unravelled about 50 species of that genus, most of which are new to science and remain to be published. The five most speciose genera listed earlier happened to be so because they benefitted from focussed and exclusive treatments. Most surveys have been conducted in a few, accessible areas of the State and several of the dense forests of the State have not been adequately explored for agarics. In short, the studies on the agarics of this region have been uneven, inadequate and therefore inconclusive. 
Table 1 Species of gilled mushrooms reported from Kerala State, India.

\begin{tabular}{|c|c|c|}
\hline Taxa & Distribution* & References \\
\hline \multicolumn{3}{|l|}{ AGARICALES } \\
\hline \multicolumn{3}{|l|}{ Agaricaceae } \\
\hline Agaricus annae Pilát & Mal & Vrinda \& Leelavathy 1996. \\
\hline Agaricus benesii (Pilát) Pilát & Thi & $\begin{array}{l}\text { Vrinda et al. 1997c, Pradeep \& Vrinda } 2007 \text { \{as Agaricus } \\
\text { squamuliferus (F.H. Møller) Pilát\} }\end{array}$ \\
\hline Agaricus bitorquis (Quél.) Sacc. & Thi & Pradeep \& Vrinda 2007. \\
\hline Agaricus campestris L. & Ern, Idu & $\begin{array}{l}\text { Bilgrami et al. 1991, Florence 2004, Pradeep \& Vrinda } \\
2007 .\end{array}$ \\
\hline Agaricus caribaeus Pegler & Thi, Way & Vrinda et al. 1999c, Mohanan, 2011. \\
\hline Agaricus carminescens Heinem. \& Gooss. & Mal & Vrinda \& Leelavathy 1996. \\
\hline Agaricus crocopeplus Berk. \& Broome & Way, Thr & Mohanan 2011. \\
\hline Agaricus endoxanthus Berk. \& Broome & Thi, Thr, Mal, Ern, Way & $\begin{array}{l}\text { Vrinda et al. 1999c, Pradeep \& Vrinda 2007, Mohanan, } \\
\text { 2011, Vrinda \& Pradeep } 2011 .\end{array}$ \\
\hline Agaricus fiardii Pegler & Thr, Mal & Mohanan 2011. \\
\hline Agaricus griseorimosus Pegler & Mal & Mohanan 2011. \\
\hline Agaricus hemilasius Berk. \& Broome & Mal & Vrinda \& Leelavathy 1996. \\
\hline Agaricus johnstonii Murrill & Thi, Pal, Mal, Ern, Thr & Vrinda et al.1997d, Mohanan 2011. \\
\hline Agaricus ochraceosquamulosus Heinem. & Way & Mohanan 2011. \\
\hline Agaricus osecanus Pilát & Thi & $\begin{array}{l}\text { Vrinda et al. 1997f, Pradeep \& Vrinda } 2007 \text { \{as Agaricus } \\
\text { nivescens (F.H. Møller) F.H. Møller\}. }\end{array}$ \\
\hline Agaricus parasilvaticus Heinem. & Mal & Vrinda \& Leelavathy 1996. \\
\hline Agaricus silvaticus Schaeff. & Thi, Mal, Thr, Way & $\begin{array}{l}\text { Pradeep \& Vrinda 2007, Varghese et al. 2010, Mohanan } \\
2011 .\end{array}$ \\
\hline Agaricus simulans Berk. & Thr & Mohanan 2011 \\
\hline Agaricus volvatulus Heinem. \& Gooss. & Thi, Mal & $\begin{array}{l}\text { Vrinda et al. 1999b, Pradeep \& Vrinda 2007, Mohanan } \\
2011 .\end{array}$ \\
\hline Chlorophyllum hortense (Murill) Vellinga & Thi, Ern, Kol & $\begin{array}{l}\text { Vrinda et al. 1999c, Pradeep \& Vrinda } 2011 \text { \{as } \\
\text { Leucoagaricus hortensis (Murrill) Pegler }\} \text {. }\end{array}$ \\
\hline Chlorophyllum molybdites (G. Mey.) Massee & Ern, Thr, Idu, Thi & $\begin{array}{l}\text { Sankaran \& Florence 1995, Florence \& Yesodharan 1997, } \\
\text { 2000, Bhavanidevi 1998, Mohanan 2003, Florence 2004, } \\
\text { Pradeep \& Vrinda 2007, Mohanan 2011, Pradeep \& Vrinda } \\
\text { 2011. }\end{array}$ \\
\hline
\end{tabular}


Mycosphere Doi 10.5943/mycosphere/4/1/6

\begin{tabular}{|c|c|c|c|}
\hline Taxa & & Distribution* & References \\
\hline & Chlorophyllum rachodes (Vittad.) Vellinga & Thi, Kan, Thr & $\begin{array}{l}\text { Bhavanidevi, 1998 as Macrolepiota rachodes (Vittad.) } \\
\text { Singer\}; Mohanan, } 2011 .\end{array}$ \\
\hline & Clarkeinda trachodes (Berk.) Singer & Ern, Mal, Thr, Kol & $\begin{array}{l}\text { Leelavathy et al. 1981, Bilgrami et al. 1991, Sankaran \& } \\
\text { Florence 1995, Florence \& Yesodharan 1997, 2000, } \\
\text { Florence 2004, Pradeep \& Vrinda 2007, Mohanan } 2011 .\end{array}$ \\
\hline & Coprinus comatus (O.F. Müll.) Pers. & Way & Varghese et al. 2010, Mohanan 2011. \\
\hline & Coprinus poliomallus Romagn. & Idu & Natarajan \& Raman 1983; Florence 2004. \\
\hline & Crucispora rhombisperma (Hongo) E. Horak & Way & $\begin{array}{l}\text { Manimohan et al. 2007; Noordeloos et al. } 2007 \text { (as } \\
\text { Panaeolina rhombisperma Hongo). }\end{array}$ \\
\hline & Cystolepiota fumosifolia (Murill) Vellinga & Thi & Kumar \& Manimohan 2009b. \\
\hline & Cystolepiota furfuracea T.K.A. Kumar \& Manim.^ & Thi & Kumar \& Manimohan 2009b. \\
\hline & Cystolepiota hemisclera (Berk. \& M.A. Curtis) Pegler & Idu & Vrinda et al. $1997 \mathrm{c}$. \\
\hline & Cystolepiota pulverulenta (Huijsman) Vellinga & Mal, Thi & $\begin{array}{l}\text { Kumar \& Manimohan 2009b, Deepa et al. } 2009 \text { as } \\
\text { Leucoagaricus pulverulentus (Huijsm.) Bon. }\}\end{array}$ \\
\hline & Echinoderma pseudoasperulum (Knudsen) Bon & Mal & $\begin{array}{l}\text { Kumar \& Manimohan 2009a \{as Lepiota pseudoasperula } \\
\text { (Knudsen) Knudsen }\} \text {. }\end{array}$ \\
\hline & Hymenagaricus alphitochrous (Berk. \& Broome) Heinem. & Mal & Heinemann \& Little Flower 1984. \\
\hline & Hymenagaricus calicutensis Heinem. \& Little Flower^^${ }^{\wedge}$ & Mal & Heinemann \& Little Flower 1984. \\
\hline & Hymenagaricus canoruber (Berk. \& Broome) Heinem. \& Little Flower & Mal & Heinemann \& Little Flower 1984. \\
\hline & Hymenagaricus cylindrocystis Heinem. \& Little Flower^^ & Mal & Heinemann \& Little Flower 1984. \\
\hline & Hymenagaricus epipastus (Berk. \& Broome) Heinem. \& Little Flower & Mal & Heinemann \& Little Flower 1984. \\
\hline & $\begin{array}{l}\text { Hymenagaricus subaeruginosus (Berk. \& Broome) Heinem. \& Little } \\
\text { Flower }\end{array}$ & Mal & Heinemann \& Little Flower 1984. \\
\hline & Lepiota alopochroa (Berk. \& Broome) Sacc. & Thr & Mohanan 2011. \\
\hline & Lepiota ananya T.K.A. Kumar \& Manim.^ & Thi & Kumar \& Manimohan 2009a. \\
\hline & Lepiota anupama T.K.A. Kumar \& Manim.^$^{\wedge}$ & Mal, Koz & Kumar \& Manimohan 2009a. \\
\hline & Lepiota apalochroa (Berk. \& Broome) Sacc. & Thi & Bhavanidevi 1998. \\
\hline & Lepiota babruka T.K.A. Kumar \& Manim.^^ & Kan & Kumar \& Manimohan 2009a. \\
\hline & Lepiota babruzalka T.K.A. Kumar \& Manim.^ $^{\wedge}$ & Koz, Mal & Kumar \& Manimohan 2009a. \\
\hline & Lepiota brevipes Murrill & Koz, Thi & $\begin{array}{l}\text { Kumar \& Manimohan 2009a (as Lepiota brevipes var. } \\
\text { distincta T.K.A. Kumar \& Manim. ^). }\end{array}$ \\
\hline & Lepiota brunneoincarnata Chod \& Mart. & Thi & Pradeep \& Vrinda 2011. \\
\hline & Lepiota castanea Quél. & Idu & Kumar \& Manimohan 2009a. \\
\hline & Lepiota citrophylla (Berk. \& Broome) Sacc. & Ern & Mohanan 2011. \\
\hline & Lepiota clypeolaria (Bull.) P. Kumm. & Way & Mohanan 2011, Pradeep \& Vrinda 2011. \\
\hline & Lepiota columbicolor (Berk. \& Broome) Sacc. & Thi & Bhavanidevi 1998. \\
\hline & Lepiota cristata (Bolton) P. Kumm. & Thi & Bhavanidevi 1998, Pradeep \& Vrinda 2011. \\
\hline & Lepiota elaiophylla Vellinga \& Huijser & Koz, Mal & Kumar \& Manimohan 2009a. \\
\hline & Lepiota erythrogramma (Berk. \& Broome) Sacc. & Mal & Mohanan 2011. \\
\hline & Lepiota erythrosticta (Berk. \& Broome) Sacc. & Mal, Ern & Kumar \& Manimohan 2009a, Mohanan 2011. \\
\hline
\end{tabular}


Mycosphere Doi 10.5943/mycosphere/4/1/6

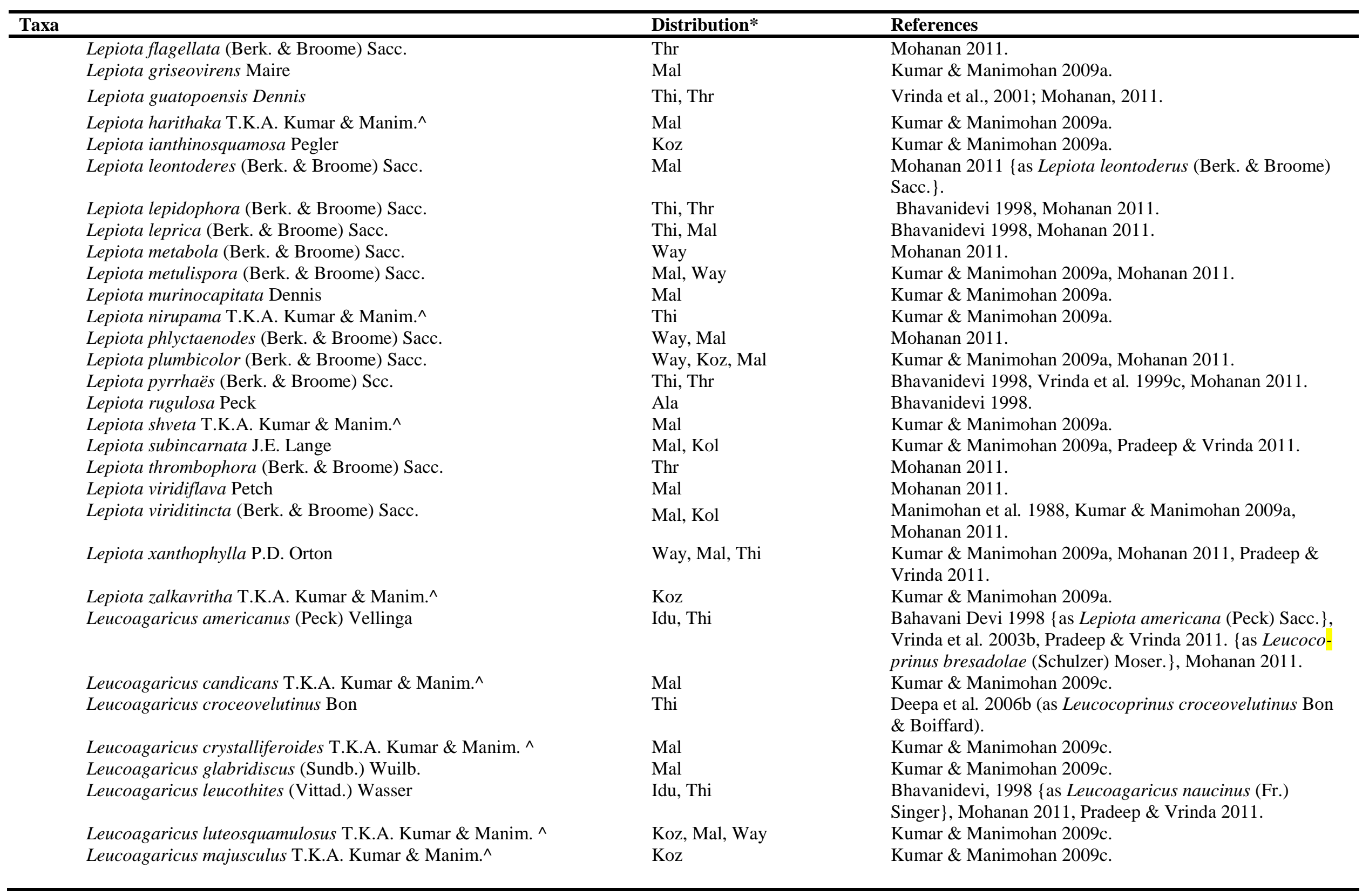




\begin{tabular}{|c|c|c|c|}
\hline Taxa & & Distribution* & References \\
\hline & Leucoagaricus melanotrichus (Malençon \& Bertault) Trimbach & Thi, Kol & $\begin{array}{l}\text { Deepa et al. } 2009 \text { (as L. melanotrichus var. septentrionalis } \\
\text { D.A. Reid and Leucoagaricus melanotrichus var. } \\
\text { fuligineobrunneus Bon \& Boiffard). }\end{array}$ \\
\hline & Leucoagaricus melanotrichus (Gray) Singer & Thi & $\begin{array}{l}\text { Vrinda et al. 2003b \{as Leucocoprinus meleagris (Gray) } \\
\text { Locq.\}. }\end{array}$ \\
\hline & Leucoagaricus quilonensis Sathe \& J.T. Daniel^^ & Kol & $\begin{array}{l}\text { Sathe \& Daniel, } 1980 \text { \{as Leucoagaricus quilonense Sathe } \\
\& \text { \& Daniel\}; Florence, } 2004 .\end{array}$ \\
\hline & Leucoagaricus rubrotinctus (Peck) Singer & $\begin{array}{l}\text { Koz, Mal, Thr, Way, Thi, } \\
\text { Idu, Kol }\end{array}$ & $\begin{array}{l}\text { Kumar \& Manimohan 2009c, Deepa et al. 2009, Mohanan } \\
\text { 2011, Pradeep \& Vrinda 2011. }\end{array}$ \\
\hline & Leucoagaricus rufosquamulosus T.K.A. Kumar \& Manim.^ & Way & Kumar \& Manimohan 2009c. \\
\hline & Leucoagaricus subflavus T.K.A. Kumar \& Manim.^${ }^{\wedge}$ & Mal & Kumar \& Manimohan 2009c. \\
\hline & Leucoagaricus sublittoralis (Kuhner ex Hora) Singer & Thi, Kol & Deepa et al. 2009. \\
\hline & ${ }^{\mathbb{I} L e u c o a g a r i c u s ~ s u l p h u r e l l u s ~(P e g l e r) ~ A k e r s ~}$ & Thi & Deepa et al. 2009. \\
\hline & Leucoagaricus tener (P.D. Orton) Bon & Thi & Deepa et al. 2009. \\
\hline & Leucoagaricus variisporus Sathe \& S.M. Kulk. & Thi & Bhavanidevi 1998. \\
\hline & Leucoagaricus viridiflavus (Petch) T.K.A. Kumar \& Manim. & Mal & Kumar \& Manimohan 2009c. \\
\hline & 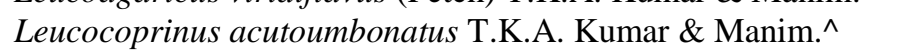 & Mal & Kumar \& Manimohan 2009c. \\
\hline & Leucocoprinus biornatus (Berk. \& Broome) Locq. & Thi & Vrinda et al. 2003b. \\
\hline & Leucocoprinus birnbaumii (Corda) Singer & $\begin{array}{l}\text { Mal, Ern, Thi, Koz, Way, } \\
\text { Idu, Kol }\end{array}$ & $\begin{array}{l}\text { Sankaran \& Florence 1995, Vrinda et al. 2003b, Florence } \\
\text { 2004, Kumar \& Manimohan 2009c, Mohanan 2011, Pradeep } \\
\text { \& Vrinda } 2011 .\end{array}$ \\
\hline & Leucocoprinus brebissonii (Godey) Locq. & Thi, Mal, Pal & $\begin{array}{l}\text { Vrinda et al. 2003b, Kumar \& Manimohan 2009c, Mohanan } \\
2011 .\end{array}$ \\
\hline & Leucocoprinus caldariorum D.A. Reid & Thi, Kol & Deepa et al. 2006a, Mohanan 2011. \\
\hline & Leucocoprinus cepistipes (Sowerby) Pat. & Thi, Way, Thr, Ern, Kol & $\begin{array}{l}\text { Bhavanidevi 1998, Vrinda et al. 2003b, Pradeep \& Vrinda } \\
2007 \text { as Leucocoprinus cepaestipes (Sow.: Fr.) Pat.\}, } \\
\text { Mohanan, } 2011 .\end{array}$ \\
\hline & Leucocoprinus cretaceus (Bull.) Locq. & Kol, Mal & Deepa et al. 2006a, Kumar \& Manimohan 2009c. \\
\hline & Leucocoprinus delicatulus T.K.A. Kumar \& Manim.^ & Mal & Kumar \& Manimohan 2009c. \\
\hline & Leucocoprinus fragilissimus (Berk. \& M.A. Curtis) Pat. & Thi, Koz, Way, Mal, Idu & $\begin{array}{l}\text { Vrinda et al. 2003b, Kumar \& Manimohan 2009c, Mohanan, } \\
2011 .\end{array}$ \\
\hline & Leucocoprinus heinemannii Migl. & Pal, Thi & Deepa et al. 2006b. \\
\hline & Leucocoprinus holospilotus (Berk. \& Broome) D.A. Reid & Thi, Mal, Koz, Thr & $\begin{array}{l}\text { Deepa et al. 2006a, Kumar \& Manimohan 2009c, Mohanan } \\
2011 \text {. }\end{array}$ \\
\hline & Leucocoprinus ianthinus (Sacc.) P. Mohr & Kan, Mal, Thi & $\begin{array}{l}\text { Deepa et al. 2006b \{as Leucocoprinus lilacinogranulosus } \\
\text { (Henn.) Locq.)\}, Kumar \& Manimohan 2009c. }\end{array}$ \\
\hline & Leucocoprinus jubilaei (Joss.) Wasser & Thi, Kol, Mal & Deepa et al. 2006b, Kumar \& Manimohan 2009c. \\
\hline
\end{tabular}


Mycosphere Doi 10.5943/mycosphere/4/1/6

\begin{tabular}{|c|c|c|c|}
\hline Taxa & & Distribution* & References \\
\hline & Leucocoprinus lacrymans T.K.A. Kumar \& Manim.^ & Koz, Mal & Kumar \& Manimohan 2004, Kumar \& Manimohan 2009c. \\
\hline & Leucocoprinus munnarensis T.K.A. Kumar \& Manim.^^ & Idu & Kumar \& Manimohan 2009c. \\
\hline & Leucocoprinus pusillus T.K.A. Kumar \& Manim.^ & Thi & Kumar \& Manimohan 2009c. \\
\hline & Leucocoprinus squamulosus (Mont.) Pegler & Thi & Vrinda et al. 2003b, Mohanan 2011. \\
\hline & Leucocoprinus straminellus (Bagl.) Narducci \& Caroti & Thi, Koz & Deepa et al. 2006a, Kumar \& Manimohan 2009c. \\
\hline & Leucocoprinus submontagnei Heinem. & Thi, Koz, Mal & Deepa et al. 2006b, Kumar \& Manimohan 2009c. \\
\hline & Leucocoprinus tenellus Pegler & Thi & Deepa et al. 2006b. \\
\hline & Leucocoprinus venezuelanus Dennis & Thi, Way, Koz & Vrinda et al. 2003b, Kumar \& Manimohan 2009c. \\
\hline & Leucocoprinus zeylanicus (Berk.) Boedijin & Thi, Mal, Ern, Thr & Vrinda et al., 1997c; Vrinda et al., 2003b; Mohanan, 2011. \\
\hline & Macrolepiota dolichaula (Berk. \& Broome) Pegler \& R.W. Rayner & Idu, Way, Thi, Kol & Pradeep \& Vrinda 2007, Mohanan 2011. \\
\hline & Macrolepiota procera (Scop.) Singer & Way, Idu, Mal, Thr & Bhavanidevi 1998, Mohanan 2011. \\
\hline & Micropsalliota alba Heinem. \& Little Flower^ & Mal & $\begin{array}{l}\text { Heinemann \& Little Flower 1983, Leelavathy \& Little } \\
\text { Flower 1986, Heinemann \& Leelavathy } 1991 .\end{array}$ \\
\hline & Micropsalliota albosericea Heinem. \& Leelavathy^ & Mal & Heinemann \& Leelavathy 1991. \\
\hline & Micropsalliota arginea (Berk. \& Broome) Pegler \& R.W. Rayner & Mal & $\begin{array}{l}\text { Leelavathy \& Little Flower 1986, Heinemann \& Leelavathy } \\
1991 .\end{array}$ \\
\hline & Micropsalliota avellanea Heinem. \& Little Flower^ & Mal & $\begin{array}{l}\text { Heinemann \& Little Flower 1983, Leelavathy \& Little } \\
\text { Flower 1986, Heinemann \& Leelavathy } 1991 .\end{array}$ \\
\hline & Micropsalliota brunneosperma (Singer) Pegler & Mal & Leelavathy \& Little Flower 1986. \\
\hline & Micropsalliota cinnamomeopallida Singer & Mal & Heinemann \& Leelavathy 1991. \\
\hline & Micropsalliota cymbispora Heinem. \& Little Flower & Mal & $\begin{array}{l}\text { Heinemann \& Little Flower 1983, Leelavathy \& Little } \\
\text { Flower 1986, Heinemann \& Leelavathy } 1991 .\end{array}$ \\
\hline & Micropsalliota cystidiosa Natarajan \& Manjula & Mal & Leelavathy \& Little Flower 1986. \\
\hline & Micropsalliota globocystis Heinem. & Mal & $\begin{array}{l}\text { Leelavathy \& Little Flower 1986, Heinemann \& Leelavathy } \\
1991 .\end{array}$ \\
\hline & Micropsalliota ianthina Heinem. & Mal & Leelavathy \& Little Flower 1986. \\
\hline & Micropsalliota malabarensis Heinem. \& Little Flower^ & Mal & $\begin{array}{l}\text { Heinemann \& Little Flower 1983, Leelavathy \& Little } \\
\text { Flower 1986, Heinemann \& Leelavathy } 1991 .\end{array}$ \\
\hline & Micropsalliota pilicystis Heinem. & Mal & $\begin{array}{l}\text { Leelavathy \& Little Flower 1986, Heinemann \& Leelavathy } \\
1991 .\end{array}$ \\
\hline
\end{tabular}


Mycosphere Doi 10.5943/mycosphere/4/1/6

\begin{tabular}{|c|c|c|c|}
\hline Taxa & & Distribution* & References \\
\hline & Micropsalliota pleurocystidiata Heinem. \& Little Flower^ & Mal & $\begin{array}{l}\text { Heinemann \& Little Flower 1983, Leelavathy \& Little } \\
\text { Flower 1986, Heinemann \& Leelavathy 1991, Mohanan } \\
\text { 2011. }\end{array}$ \\
\hline & Micropsalliota plumaria (Berk. \& Broome) Höhn. & Mal & Heinemann \& Leelavathy 1991. \\
\hline & Micropsalliota pseudovolvulata Höhn. & Mal & Leelavathy \& Little Flower 1986. \\
\hline & Micropsalliota pudica Heinem. \& Leelav. ^ & Mal & Heinemann \& Leelavathy 1991. \\
\hline & Micropsalliota pulverulenta Heinem. \& Leelav. ^^ & Mal & Heinemann \& Leelavathy 1991. \\
\hline & Micropsalliota repanda Heinem. & Mal & Leelavathy \& Little Flower 1986. \\
\hline & Micropsalliota subalba Heinem. \& Little Flower^ & Mal & $\begin{array}{l}\text { Heinemann \& Little Flower 1983, Leelavathy \& Little } \\
\text { Flower 1986, Heinemann \& Leelavathy } 1991 .\end{array}$ \\
\hline & Xanthagaricus brunneolus Little Flower, Hosag. \& T.K. Abraham^ & Mal & Little Flower et al., 1997. \\
\hline & $\begin{array}{l}\text { Xanthagaricus chrysosporus (Heinem. \& Little Flower) Little Flower, } \\
\text { Hosag. \& T.K. Abraham }\end{array}$ & Mal & $\begin{array}{l}\text { Heinemann \& Little Flower } 1984 \text { (as Hymenagaricus } \\
\text { chrysosporus Heinem. \& Little Flower^), Little Flower et } \\
\text { al.1997. }\end{array}$ \\
\hline & $\begin{array}{l}\text { Xanthagaricus erinaceus (Heinem. \& Little Flower) Little Flower, } \\
\text { Hosag. \& T.K. Abraham }\end{array}$ & Mal & $\begin{array}{l}\text { Heinemann \& Little Flower } 1984 \text { (as Hymenagaricus } \\
\text { erinaceus Heinem. \& Little Flower^), Little Flower et al. } \\
1997 .\end{array}$ \\
\hline & $\begin{array}{l}\text { Xanthagaricus flavidorufus (Berk. \& Broome) Little Flower, Hosag. \& } \\
\text { T.K. Abraham }\end{array}$ & Mal & $\begin{array}{l}\text { Heinemann \& Little Flower } 1984 \text { \{as Hymenagaricus } \\
\text { flavidorufus (Berk. \& Broome) Heinem. \& Little Flower\}, } \\
\text { Little Flower et al., } 1997 .\end{array}$ \\
\hline & $\begin{array}{l}\text { Xanthagaricus globisporus (Heinem. \& Little Flower) Little Flower, } \\
\text { Hosag. \& T.K. Abraham }\end{array}$ & Mal & $\begin{array}{l}\text { Heinemann \& Little Flower, } 1984 \text { (as Hymenagaricus } \\
\text { globisporus Heinem. \& Little Flower }{ }^{\wedge} \text { ), Little Flower et } \\
\text { al.1997. }\end{array}$ \\
\hline & $\begin{array}{l}\text { Xanthagaricus gracilis (Heinem. \& Little Flower) Little Flower, Hosag. } \\
\& \text { T.K. Abraham }\end{array}$ & Mal & $\begin{array}{l}\text { Heinemann \& Little Flower } 1984 \text { (as Hymenagaricus } \\
\text { gracilis Heinem. \& Little Flower^), Little Flower et al.1997. }\end{array}$ \\
\hline & $\begin{array}{l}\text { Xanthagaricus luteolosporus (Heinem. \& Little Flower) Little Flower, } \\
\text { Hosag. \& T.K. Abraham }\end{array}$ & Mal & $\begin{array}{l}\text { Heinemann \& Little Flower } 1984 \text { (as Hymenagaricus } \\
\text { luteolosporus Heinem. \& Little Flower^), Little Flower et al. } \\
\text { 1997. }\end{array}$ \\
\hline & $\begin{array}{l}\text { Xanthagaricus myriostictus (Berk. \& Broome) Little Flower, Hosag. \& } \\
\text { T.K. Abraham }\end{array}$ & Mal & $\begin{array}{l}\text { Heinemann \& Little Flower } 1984 \text { \{as Hymenagaricus } \\
\text { myriostictus (Berk. \& Broome) Heinem. \& Little Flower }\} \\
\text { Little Flower et al. } 1997 .\end{array}$ \\
\hline & Xanthagaricus nanus Little Flower, Hosag. \& T.K. Abraham^^ & Mal & Little Flower et al. 1997. \\
\hline
\end{tabular}




\begin{tabular}{|c|c|c|}
\hline Taxa & Distribution* & References \\
\hline $\begin{array}{l}\text { Xanthagaricus rubescens (Heinem. \& Little Flower) Little Flower, } \\
\text { Hosag. \& T.K. Abraham }\end{array}$ & Mal & $\begin{array}{l}\text { Heinemann \& Little Flower } 1984 \text { (as Hymenagaricus } \\
\text { rubescens Heinem. \& Little Flower^), Little Flower et al. } \\
1997 .\end{array}$ \\
\hline $\begin{array}{l}\text { Xanthagaricus subepipastus (Heinem. \& Little Flower) Little Flower, } \\
\text { Hosag. \& T.K. Abraham }\end{array}$ & Mal & $\begin{array}{l}\text { Heinemann \& Little Flower } 1984 \text { (as Hymenagaricus } \\
\text { subepipastus Heinem. \& Little Flower^), Little Flower et al. } \\
1997 .\end{array}$ \\
\hline $\begin{array}{l}\text { Xanthagaricus viridulus (Heinem. \& Little Flower) Little Flower, } \\
\text { Hosag. \& T.K. Abraham }\end{array}$ & Mal & $\begin{array}{l}\text { Heinemann \& Little Flower } 1984 \text { (as Hymenagaricus } \\
\text { viridulus Heinem. \& Little Flower^), Little Flower et al. } \\
\text { 1997. }\end{array}$ \\
\hline \multicolumn{3}{|r|}{ 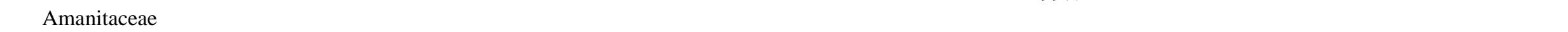 } \\
\hline Amanita angustilamellata (Höhn.) Boedijn & Thi, Way, Mal, Ern, Kol & Vrinda et al. 1997f, Pradeep \& Vrinda 2010, Mohanan 2011. \\
\hline Amanita aureofloccosa Bas & Thi, Ern & $\begin{array}{l}\text { Vrinda et al. 1997f, Pradeep \& Vrinda 2007, Pradeep \& } \\
\text { Vrinda 2010, Mohanan } 2011 .\end{array}$ \\
\hline Amanita bharatensis Sathe \& J.T. Daniel^ & Idu & Sathe \& Daniel 1980, Florence 2004. \\
\hline Amanita bisporigera G.F. Atk. & Way & Mohanan 2011. \\
\hline Amanita ceciliae (Berk. \& Broome) Bas & Way & Mohanan, 2011. \\
\hline Amanita elata (Massee) Corner \& Bas & Thi, Kol, Way & Pradeep \& Vrinda 2010, Mohanan 2011. \\
\hline Amanita griseofarinosa Hongo & Thi, Way, Ern, Mal & Pradeep \& Vrinda 2010, Mohanan 2011. \\
\hline Amanita hemibapha (Berk. \& Broome) Sacc. & Thi, Mal, Way & $\begin{array}{l}\text { Vrinda et al. 2005a, Pradeep \& Vrinda 2007, Pradeep \& } \\
\text { Vrinda 2010, Mohanan } 2011 .\end{array}$ \\
\hline Amanita magniverrucata Thiers \& Ammirati & Thi & Pradeep \& Vrinda 2010 \\
\hline Amanita muscaria (L.) Lam & Idu & $\begin{array}{l}\text { Pradeep \& Vrinda 2007, Mohanan 2011, Pradeep \& Vrinda } \\
2011 .\end{array}$ \\
\hline Amanita phalloides (Vaill. ex Fr.) Link & Thi & $\begin{array}{l}\text { Vrinda et al. 2005b, Pradeep \& Vrinda 2007, Pradeep \& } \\
\text { Vrinda } 2011 .\end{array}$ \\
\hline Amanita porphyria Alb. \& Schwein & Ern, Mal & Mohanan 2011. \\
\hline Amanita solitaria (Bull.) Fr. & Thi & $\begin{array}{l}\text { Bhavanidevi \& Nair 1983, Florence 2004, Pradeep \& Vrinda } \\
2011 .\end{array}$ \\
\hline Amanita volvata (Peck) Lloyd & Thi & Pradeep \& Vrinda 2010, Pradeep \& Vrinda 2011. \\
\hline Amanita verna (Bull.) Lam. & Thi & Pradeep \& Vrinda 2011. \\
\hline Catatrama costaricensis Franco-Mol. & Ern, Pat & Vrinda et al. 2000c, Pradeep \& Vrinda 2007. \\
\hline Limacella guttata (Pers.) Konrad \& Maubl. & Mal, Ern & Mohanan 2011. \\
\hline Limacella myxodictyon (Berk. \& Broome) Pegler & Ern, Mal & Manimohan et al. 1988. \\
\hline $\begin{array}{l}\text { Limacella quilonensis Sathe \& J.T. Daniel^ } \\
\text { Bolbitiaceae }\end{array}$ & Kol & Sathe \& Daniel 1980, Florence 2004. \\
\hline Bolbitius coprophilus (Peck) Hongo & Thr, Kas & Thomas et al. 2001, Manimohan et al. 2007. \\
\hline
\end{tabular}


Mycosphere Doi 10.5943/mycosphere/4/1/6

\begin{tabular}{|c|c|c|}
\hline Taxa & Distribution* & References \\
\hline Bolbitius fissus Berk. \& Broome & Mal, Thr & Mohanan 2011. \\
\hline Bolbitius titubans (Bull.) Fr. & Kol, Thr & Mohanan 2011. \\
\hline Conocybe brunneoaurantiaca K.A. Thomas, Hauskn. \& Manim. ^ & Mal & Thomas et al. 2001, Manimohan et al. 2007. \\
\hline Conocybe crispa (Longyear) Singer & Kol & Mohanan 2011. \\
\hline Conocybe juniana (Velen.) Hauskn. \& Svrček & Mal & $\begin{array}{l}\text { Watling et al. } 1988 \text { (as Conocybe magnicapitata P.D. } \\
\text { Orton). }\end{array}$ \\
\hline Conocybe magnispora (Murrill) Singer & Mal & Watling et al. 1988. \\
\hline Conocybe mesospora Kühner ex Watling & Mal & Watling et al. 1988. \\
\hline Conocybe ochracea (Kühner) Singer & Mal & Mohanan 2011. \\
\hline Conocybe pilosella (Pers.) Kuhner & Idu & Natarajan \& Raman 1983, Florence 2004. \\
\hline Conocybe pulchella (Velen.) Hauskn. \& Svrček & Mal & $\begin{array}{l}\text { Watling et al. } 1988 \text { (as Conocybe pseudopilosella Kühner \& } \\
\text { Watling). }\end{array}$ \\
\hline Conocybe pseudopubescens K.A. Thomas, Hauskn. \& Manim. ${ }^{\wedge}$ & Way & Thomas et al. 2001. \\
\hline Conocybe pubescens (Gillet) Kühner & Mal, Thr & Watling et al. 1988, Mohanan 2011. \\
\hline Conocybe radicans K.A. Thomas, Hauskn. \& Manim. ${ }^{\wedge}$ & Way & Thomas et al. 2001. \\
\hline Conocybe rickenii (Jul. Schäff.) Kühner & Ern & Mohanan 2011. \\
\hline Conocybe semiglobata Kühner \& Watling & Mal & Watling et al., 1988. \\
\hline Conocybe siennophylla (Berk. \& Broome) Singer & Mal & Watling et al. 1988, Thomas et al. 2001. \\
\hline Conocybe solitaria K. A. Thomas, Hauskn. \& Manim. ${ }^{\wedge}$ & Mal & Thomas et al., 2001. \\
\hline Conocybe tenera (Schaeff.) Fayod & Mal & Mohanan 2011. \\
\hline Conocybe velutipes (Velen.) Hauskn. \& Svrček & Mal & $\begin{array}{l}\text { Watling et al. } 1988 \text { (as Conocybe kuehneriana Singer), } \\
\text { Thomas et al. } 2001 \text { as Conocybe aff. velutipes (Velen.) } \\
\text { Hauskn. \& Svrček\}. }\end{array}$ \\
\hline Conocybe volvata K.A. Thomas, Hauskn. \& Manim. ${ }^{\wedge}$ & Thr & Thomas et al. 2001, Manimohan et al. 2007. \\
\hline Conocybe zeylanica (Petch) Boedijn & Kan, Mal, Thr, Way & Watling et al. 1988, Thomas et al. 2001, Mohanan 2011. \\
\hline Galerella plicatella (Peck) Singer & Mal & Thomas et al. 2001. \\
\hline 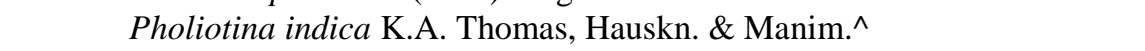 & Way, Idu & Thomas et al. 2001, Manimohan et al. 2007, Mohanan 2011. \\
\hline Pholiotina utricystidiata Enderle \& H.-J. Hübner & Idu & Thomas et al. 2001. \\
\hline \multicolumn{3}{|l|}{ Cortinariaceae } \\
\hline Anamika indica K.A. Thomas, Peintner, M.M. Moser \& Manim.^^ & Way, Idu & Thomas et al. 2002b, Mohanan 2011. \\
\hline Cortinarius cinnabarinus Fr. & Thi & Bhavanidevi \& Nair 1983, Florence 2004. \\
\hline $\begin{array}{l}\text { Cortinarius conopileus K.A. Thomas, M.M. Moser, Peintner \& } \\
\text { Manim.^ }^{\wedge}\end{array}$ & Way & Peintner et al. 2003. \\
\hline 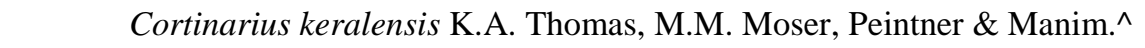 & Way & Peintner et al. 2003. \\
\hline Cortinarius palmicola Sathe \& J.T. Daniel^ ${ }^{\wedge}$ & Kol & $\begin{array}{l}\text { Sathe \& Daniel } 1980 \text { as Cortinarius palmicolous Sathe \& } \\
\text { Daniel }\} \text {, Florence } 2004 .\end{array}$ \\
\hline
\end{tabular}


Mycosphere Doi 10.5943/mycosphere/4/1/6

\begin{tabular}{|c|c|c|}
\hline Taxa & Distribution* & References \\
\hline $\begin{array}{l}\text { Cortinarius phlegmophorus K.A. Thomas, M.M. Moser, Peintner \& } \\
\text { Manim. } \wedge\end{array}$ & Way & Peintner et al. 2003. \\
\hline Cortinarius pholideus (Lilj.) Fr. & Way & Mohanan 2011. \\
\hline $\begin{array}{l}\text { Descolea maculata Bougher } \\
\text { Entolomataceae }\end{array}$ & Idu & Thomas et al. 2001 (treated in Bolbitiaceae). \\
\hline Alboleptonia aurantia Manim. \& Leelav. ^ & Mal & $\begin{array}{l}\text { Manimohan \& Leelavathy 1988b, Manimohan et al. } 1995 \text { (= } \\
\text { Entoloma aurantium (Manim. \& Leelav.) Manim., A.V. } \\
\text { Joseph \& Leelav.). }\end{array}$ \\
\hline Alboleptonia graveolens Manim. \& Leelav. ^ & Mal, Way & $\begin{array}{l}\text { Manimohan \& Leelavathy 1988, Manimohan et al. 1995(= } \\
\text { Entoloma theekshnagandhum Manim., A.V. Joseph \& } \\
\text { Leelav.). }\end{array}$ \\
\hline Alboleptonia stylophora (Berk. \& Broome) Pegler & Mal & Leelavathy \& Little Flower 1981. \\
\hline Clitopilus peri Berk. \& Broome & Thi & $\begin{array}{l}\text { Sathe \& Daniel 1980, Florence } 2004 \text { (as C. peri var. } \\
\text { megaforma Sathe \&J.T. Daniel). }\end{array}$ \\
\hline Entoloma abortivum (Berk. \& M.A. Curtis) Donk & Thi & $\begin{array}{l}\text { Bhavanidevi \& Nair 1983, Florence } 2004 \text { \{as Rhodophyllus } \\
\text { abortivus (Berk.\& M.A.Curtis)Singer\}. }\end{array}$ \\
\hline Entoloma albidoquadratum Manim. \& Noordel. ${ }^{\wedge}$ & Way & Manimohan et al. 2006, Mohanan 2011. \\
\hline Entoloma allocybesimilis Manim. \& Noordel. ^ & Way & Manimohan et al. 2006, Mohanan 2011. \\
\hline Entoloma anamikum Manim., A.V. Joseph \& Leelav.^ & Pal, Mal & $\begin{array}{l}\text { Manimohan et al. 1995, Manimohan et al. 2006, Mohanan } \\
2011 .\end{array}$ \\
\hline $\begin{array}{l}\text { Entoloma aurantium (Manim. \& Leelav.) Manim., A.V. Joseph \& } \\
\text { Leelav. }\end{array}$ & Mal & Manimohan et al. 1995, Manimohan et al. 2006. \\
\hline Entoloma brihadum Manim., A.V. Joseph \& Leelav.^ & Way, Kol & $\begin{array}{l}\text { Manimohan et al., 1995; Manimohan et al., 2006; Mohanan, } \\
2011 .\end{array}$ \\
\hline Entoloma brunneoquadratum Manim. \& Noordel. ^ & Way, Mal & Manimohan et al. 2006, Mohanan 2011. \\
\hline Entoloma calliviolaceum Manim. \& Noordel. ${ }^{\wedge}$ & Way, Idu, Mal & Manimohan et al. 2006, Mohanan 2011. \\
\hline Entoloma carneum Manim. \& Noordel.^ & Kan & $\begin{array}{l}\text { Manimohan et al. 2002, Manimohan et al. } 2006\{=E \text {. } \\
\text { indocarneum Manim. \& Noordel. }\} \text {. }\end{array}$ \\
\hline Entoloma dysthales (Peck) Sacc. & Pal & $\begin{array}{l}\text { Manimohan et al. } 2006 \text { as E. dysthales var. keralense } \\
\text { Manim. \& Noordel.^^\}. }\end{array}$ \\
\hline Entoloma flavidum (Massee) Corner \& E. Horak & Way & $\begin{array}{l}\text { Manimohan et al. 1995, Manimohan et al. 2006, Mohanan } \\
2011 .\end{array}$ \\
\hline Entoloma gnaphalodes (Berk. \& Broome) E. Horak & Mal, Way & $\begin{array}{l}\text { Manimohan et al. 1995, Manimohan et al. 2006, Mohanan, } \\
2011 .\end{array}$ \\
\hline Entoloma gnophodes (Berk. \& Broome) E. Horak & Mal & Manimohan et al. 1995, Manimohan et al. 2006. \\
\hline Entoloma granuliferum E. Horak & Thi & Vrinda et al. 1996a (as Entoloma granulifer E. Horak). \\
\hline Entoloma griseolazulinum Manim. \& Noordel. ^ & Kan, Way, Pal & Manimohan et al. 2006, Mohanan 2011. \\
\hline Entoloma griseoviolascens Manim. \& Noordel. ^ & Mal, Kol, Mal & Manimohan et al. 2006, Mohanan 2011. \\
\hline
\end{tabular}


Mycosphere Doi 10.5943/mycosphere/4/1/6

\begin{tabular}{|c|c|c|c|}
\hline Taxa & & Distribution* & References \\
\hline & 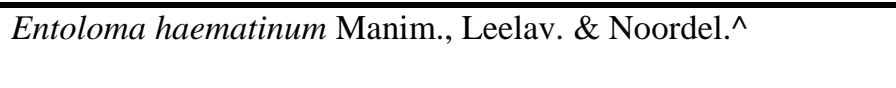 & Mal, Kol & $\begin{array}{l}\text { Manimohan et al. 2002, Manimohan et al. 2006, Mohanan } \\
2011 .\end{array}$ \\
\hline & Entoloma hyalodepas (Berk. \& Broome) E. Horak & Way & $\begin{array}{l}\text { Manimohan et al. 1995, Manimohan et al. 2006, Mohanan } \\
2011 .\end{array}$ \\
\hline & Entoloma indoviolaceum Manim. \& Noordel. ${ }^{\wedge}$ & Mal, Thi & Manimohan et al. 2006, Mohanan 2011. \\
\hline & Entoloma infundibuliforme Petch & Mal & Manimohan et al. 1995, Manimohan et al. 2006. \\
\hline & Entoloma keralense Manim. \& Noordel. ${ }^{\wedge}$ & Mal, Way & Manimohan et al. 2006, Mohanan 2011. \\
\hline & Entoloma lomapadum Manim., A.V. Joseph \& Leelav.^ & Mal & $\begin{array}{l}\text { Manimohan et al. 1995, Manimohan et al. 2006, Mohanan } \\
2011 .\end{array}$ \\
\hline & Entoloma maleolens E. Horak & Mal & Manimohan et al. 1995, Manimohan et al. 2006. \\
\hline & Entoloma mridulum Manim., A.V. Joseph \& Leelav.^ & Mal, Way & $\begin{array}{l}\text { Manimohan et al. 1995, Manimohan et al. 2006, Mohanan } \\
2011 .\end{array}$ \\
\hline & Entoloma niranjanum Manim., A.V. Joseph \& Leelav.^ & Mal & $\begin{array}{l}\text { Manimohan et al. 1995, Manimohan et al. 2006, Mohanan } \\
2011 .\end{array}$ \\
\hline & Entoloma nirupamum Manim., A.V. Joseph \& Leelav.^${ }^{\wedge}$ & Mal, Way & $\begin{array}{l}\text { Manimohan et al. 1995, Manimohan et al. 2006, Mohanan } \\
2011 .\end{array}$ \\
\hline & Entoloma nubilum Manim., Leelav. \& Noordel.^ & Mal & Manimohan et al. 2002, Manimohan et al. 2006. \\
\hline & Entoloma perflavidum Manim. \& Noordel. ${ }^{\wedge}$ & Kgd, Idu & Manimohan et al. 2006, Mohanan 2011. \\
\hline & Entoloma perinfundibuliforme Manim. \& Noordel. ${ }^{\wedge}$ & Way, Kol & Manimohan et al. 2006, Mohanan 2011. \\
\hline & Entoloma pervelutinum E. Horak & Mal, Way, Kol & $\begin{array}{l}\text { Manimohan et al. 1995, Manimohan et al. 2006, Mohanan } \\
2011 .\end{array}$ \\
\hline & Entoloma proprium E. Horak & Mal, Way, Kol & $\begin{array}{l}\text { Manimohan et al. 1995, Manimohan et al. 2006, Mohanan } \\
2011 \text {. }\end{array}$ \\
\hline & Entoloma pruinosocutis Manim. \& Noordel. ^ & Mal & Manimohan et al. 2006 \\
\hline & Entoloma rugosopruinatum Corner \& E. Horak & Mal, Way & $\begin{array}{l}\text { Manimohan et al. 1995, Manimohan et al. 2006, Mohanan } \\
2011 .\end{array}$ \\
\hline & Entoloma serrulatum (Fr.) Hesler & Mal, Way & $\begin{array}{l}\text { Manimohan et al., 1995; Manimohan et al., 2006; Mohanan, } \\
2011 .\end{array}$ \\
\hline & Entoloma shwethum Manim., A.V. Joseph \& Leelav.^ & Mal, Way & $\begin{array}{l}\text { Manimohan et al. 1995, Manimohan et al. 2006, Mohanan } \\
2011 .\end{array}$ \\
\hline & Entoloma stylophorum (Berk. \& Broome) Sacc. & Mal & Manimohan et al. 1995, Manimohan et al. 2006. \\
\hline & Entoloma tectonicola Manim. \& Noordel. ^ & Mal, Way & Manimohan et al. 2006, Mohanan 2011. \\
\hline & Entoloma testaceostrigosum Manim. \& Noordel. ^ & Way & Manimohan et al. 2006. \\
\hline & Entoloma theekshnagandhum Manim., A.V. Joseph \& Leelav.^ & Mal, Thi, Way & $\begin{array}{l}\text { Manimohan et al. 1995, Manimohan et al. 2006, Mohanan, } \\
2011 .\end{array}$ \\
\hline & Entoloma vanajum Manim., A.V. Joseph \& Leelav.^ & Way, Mal & $\begin{array}{l}\text { Manimohan et al. 1995, Manimohan et al. 2006, Mohanan } \\
2011 .\end{array}$ \\
\hline
\end{tabular}


Mycosphere Doi 10.5943/mycosphere/4/1/6

\begin{tabular}{|c|c|c|}
\hline Taxa & Distribution* & References \\
\hline Entoloma violaceovillosum Manim. \& Noordel. ${ }^{\wedge}$ & Way & Manimohan et al. 2006. \\
\hline Pouzarella fulvolanata (Berk. \& Broome) Mazzer & Thi & Vrinda et al. 1996a. \\
\hline Pouzaromyces lasius (Berk. \& Broome) Pegler & Mal & $\begin{array}{l}\text { Leelavathy \& Little Flower 1981, Bilgrami et al. 1991, } \\
\text { Florence } 2004 .\end{array}$ \\
\hline Pouzaromyces myodermus (Berk. \& Broome) Pegler & Mal & $\begin{array}{l}\text { Leelavathy \& Little Flower 1981, Bilgrami et al. 1991, } \\
\text { Florence } 2004 .\end{array}$ \\
\hline Rhodocybe caelatoidea Dennis & $\mathrm{Pal}$ & Mohanan 2011. \\
\hline Rhodocybe collybioides Singer & Thi & Vrinda et al. 2000b. \\
\hline Rhodocybe nitellina (Fr.) Singer & Thi & Vrinda et al. 2000b. \\
\hline Rhodocybe retroflexa (Berk. \& Broome) Pegler & Thi & Mohanan 2011. \\
\hline Rhodocybe subgilva (Berk. \& Broome) Pegler & Mal & Manimohan et al. 1988. \\
\hline \multicolumn{3}{|l|}{ Hydnangiaceae } \\
\hline Laccaria amethystina Cooke & Kol & Mohanan 2011. \\
\hline Laccaria fraterna (Sacc.) Pegler & Idu & Mohanan 2011. \\
\hline Laccaria laccata (Scop.) Cooke & Kol, Mal, Way, Ern, Thi & $\begin{array}{l}\text { Pradeep \& Vrinda 2010, Varghese et al. 2010, Mohanan } \\
2011 .\end{array}$ \\
\hline Laccaria ohiensis (Mont.) Singer & Idu & Mohanan 2011 \\
\hline \multicolumn{3}{|l|}{ Hygrophoraceae } \\
\hline Camarophyllus umbrinus (Dennis) Singer ex Pegler & Thr & Mohanan 2011. \\
\hline Hygrocybe acutoconica (Clem.) Singer & Thi, Mal, Way & $\begin{array}{l}\text { Vrinda et al. } 1995 \text { as Hygrocybe konradii R. Haller Aar. (= } \\
\text { H. acutoconica var. konradii (R. Haller Aar.) Boertm.)\}, } \\
\text { Leelavathy et al. 2006, Mohanan } 2011 \text {. }\end{array}$ \\
\hline Hygrocybe alwisii (Berk. \& Broome) Pegler & Thi, Mal, Way, Pal, Kol & $\begin{array}{l}\text { Vrinda et al. 1996c, Leelavathy et al. 2006, Pradeep \& } \\
\text { Vrinda 2007, Mohanan } 2011\end{array}$ \\
\hline Hygrocybe anisa (Berk. \& Broome) Pegler & Kol & Mohanan, 2011. \\
\hline Hygrocybe apala (Berk. \& Broome) Pegler \& R.W. Rayner & Mal, Way & $\begin{array}{l}\text { Leelavathy et al. 2006, Mohanan } 2011 \text { (Hygrocybe apala } \\
\text { var. indica Leelav., Manim. \& Arnolds }{ }^{\wedge} \text { ). }\end{array}$ \\
\hline Hygrocybe astatogala (R. Heim) Heinem. & Mal, Way, Thr & Leelavathy et al. 2006, Mohanan 2011. \\
\hline Hygrocybe atrosquamosa Pegler & Thi & Vrinda et al. $1997 \mathrm{~d}$. \\
\hline Hygrocybe aurantia Murrill & Mal & Leelavathy et al. 2006. \\
\hline Hygrocybe aurantioalba Leelav., Manim. \& Arnolds^ & Mal, Idu, Kol & Leelavathy et al. 2006, Mohanan 2011. \\
\hline Hygrocybe aurantiocephala Leelav., Manim. \& Arnolds^ & Mal, Way & Leelavathy et al. 2006, Mohanan 2011. \\
\hline
\end{tabular}


Mycosphere Doi 10.5943/mycosphere/4/1/6

\begin{tabular}{|c|c|c|c|}
\hline Taxa & & Distribution* & References \\
\hline & Hygrocybe batistae Singer & Thi, Kol & Vrinda et al. 2009. \\
\hline & Hygrocybe brunneosquamulosa Leelav., Manim. \& Arnolds^ & Mal, Kol, Way & Leelavathy et al. 2006, Mohanan 2011. \\
\hline & Hygrocybe caespitosa Murrill & Thi & Vrinda et al. 1995. \\
\hline & Hygrocybe cantharellus (Schwein.) Murrill & Thi, Mal & Vrinda et al. 1995, Leelavathy et al. 2006, Mohanan 2011. \\
\hline & Hygrocybe cinerascens (Berk. \& Broome) Pegler & Mal, Thr, Ern & Leelavathy et al. 2006, Mohanan 2011. \\
\hline & Hygrocybe coccinea (Schaeff.) P. Kumm. & Idu & Mohanan 2003, Florence 2004. \\
\hline & Hygrocybe conica (Schaeff.) P. Kumm. & Thi, Mal, Thr & $\begin{array}{l}\text { Sankaran \& Florence 1995, Vrinda et al. 1995, Florence } \\
2004 \text { \{as Hygrocybe conica var. conica (Schaeff.) P. } \\
\text { Kumm.\}, Leelavathy et al. } 2006 \text { \{as Hygrocybe conica var. } \\
\text { conicopalustris (R. Haller Aar.) Heinem\}, Pradeep \& } \\
\text { Vrinda } 2011 .\end{array}$ \\
\hline & Hygrocybe corallina Leelav., Manim. \& Arnolds^ ${ }^{\wedge}$ & Mal & Leelavathy et al. 2006. \\
\hline & Hygrocybe cuspidata (Peck) Murrill & Mal, Way & $\begin{array}{l}\text { Leelavathy et al. } 2006 \text { \{as Hygrocybe acutoconica var. } \\
\text { cuspidata (Peck) Arnolds\}, Mohanan } 2011 .\end{array}$ \\
\hline & Hygrocybe deceptiva (A.H. Sm. \& Hesler) Leelav., Manim. \& Arnolds & Mal & Leelavathy et al. 2006, Mohanan 2011. \\
\hline & Hygrocybe diversicolor (Petch) Pegler & Kol & Vrinda et al. 2009, Mohanan 2011. \\
\hline & Hygrocybe erinacea (Pat.) Singer & Thi & Vrinda et al. 1997d. \\
\hline & Hygrocybe firma (Berk. \& Broome) Singer & Kan & Mohanan 2011. \\
\hline & Hygrocybe globispora Leelav., Manim. \& Arnolds^ & Mal & Leelavathy et al. 2006 \\
\hline & Hygrocybe gregaria Leelav., Manim. \& Arnolds^ & Mal, Way & Leelavathy et al. 2006, Mohanan 2011. \\
\hline & Hygrocybe griseoalbida Leelav., Manim. \& Arnolds^ & Mal & Leelavathy et al. 2006. \\
\hline & Hygrocybe hypohaemacta (Corner) Pegler & Thi & Vrinda et al. 1996a. \\
\hline & Hygrocybe insipida (J.E. Lange) M.M. Moser & Mal & Leelavathy et al. 2006. \\
\hline & Hygrocybe keralensis Leelav., Manim. \& Arnolds^ ${ }^{\wedge}$ & Way & Leelavathy et al. 2006, Mohanan 2011. \\
\hline & Hygrocybe lobatospora Leelav., Manim. \& Arnolds ${ }^{\wedge}$ & Way & Leelavathy et al., 2006. \\
\hline & Hygrocybe martinicensis Pegler \& Fiard & Thi & Vrinda et al. 2009. \\
\hline & Hygrocybe mexicana Singer & Mal, Idu & Leelavathy et al. 2006, Mohanan 2011. \\
\hline & Hygrocybe nigrescens (Quél.) Kühner & Thi & Vrinda et al. 1995. \\
\hline & Hygrocybe nivosa Berk. \& Broome) Leelav., Manim. \& Arnolds^ & Mal, Way, Thr & $\begin{array}{l}\text { Leelavathy et al. 2006, Mohanan } 2011\{\text { as } H . \text { nivosa var. } \\
\text { nivosa (Berk. \& Broome) Leelav., Manim. \& Arnolds^ and } \\
\left.\text { H. nivosa var. pallidolutea Leelav., Manim. \& Arnolds }{ }^{\wedge}\right\} .\end{array}$ \\
\hline
\end{tabular}


Mycosphere Doi 10.5943/mycosphere/4/1/6

\begin{tabular}{|c|c|c|}
\hline Taxa & Distribution* & References \\
\hline Hygrocybe ortoniana Bon & Mal, Kol & Leelavathy et al. 2006, Mohanan 2011. \\
\hline Hygrocybe parvispora T.K. Abraham, K.B. Vrinda \& C.K. Pradeep ${ }^{\wedge}$ & Thi, Kol & Abraham et al. 1996, Pradeep \& Vrinda 2007. \\
\hline Hygrocybe parvula (Peck) Murrill & Way & Leelavathy et al. 2006, Mohanan 2011. \\
\hline Hygrocybe pratensis (Fr.) Murrill & Way, Kol & Leelavathy et al. 2006, Mohanan 2011. \\
\hline Hygrocybe punicea (Fr.) P. Kumm. & Thi & Pradeep et al. 1996b, Pradeep \& Vrinda 2007. \\
\hline Hygrocybe smaragdina Leelav., Manim. \& Arnolds^ ${ }^{\wedge}$ & Mal, Kol & Leelavathy et al. 2006, Mohanan 2011. \\
\hline Hygrocybe subminutula Murrill & Thi & Vrinda et al. 1995. \\
\hline Hygrocybe viridula Lodge \& Pegler & Thi & Vrinda et al. 1995. \\
\hline \multicolumn{3}{|l|}{ Inocybaceae } \\
\hline Astrosporina hydrocybiformis Corner \& E. Horak & Thi, Ern & Vrinda et al. 1999b. \\
\hline Astrosporina punctatosquamosa $\mathrm{E}$. Horak & Thi & Vrinda et al. 2001. \\
\hline Crepidotus calolepis (Fr.) P. Karst. & Ern & Mohanan 2011. \\
\hline Crepidotus citrinus Petch & Thi, Idu & Vrinda et al. 2000a, Mohanan 2011. \\
\hline Crepidotus cystidiosus Hesler \& A.H. Sm. & Idu & Mohanan 2011. \\
\hline Crepidotus epicrocinus Berk. \& Broome & Idu & Mohanan 2011. \\
\hline Crepidotus grumosopilosus (Berk. \& Broome) Sacc. & Idu & Mohanan 2011. \\
\hline Crepidotus melleus (Berk. \& Broome) Petch & Pal & Mohanan 2011. \\
\hline Crepidotus nephrodes (Berk. \& M.A. Curtis) Sacc. & Mal & Mohanan 2011. \\
\hline Crepidotus pezizula ( Berk. \& Br.) Sacc. & Thi & Vrinda et al. 2000a. \\
\hline Crepidotus reversus Berk. \& Broome & Kol & Mohanan 2011. \\
\hline Crepidotus uber (Berk. \& M.A. Curtis) Sacc. & Pal & Mohanan 2011. \\
\hline Inocybe acuta Boud & Thi & Vrinda et al. $1997 \mathrm{~b}$. \\
\hline Inocybe antillana Pegler & Way & Mohanan 2011. \\
\hline Inocybe assimilata Britzelm. & Thi & Vrinda et al., 1997b (as Inocybe umbrina Masses) \\
\hline Inocybe bongardii (Weinm.) Quél. & Ern & Bilgrami et al. 1991, Florence 2004. \\
\hline Inocybe crassicystidiata Pegler & Ern & Mohanan 2011. \\
\hline Inocybe cutifracta Petch & Mal, Thr, Kol, Ern, Thi & $\begin{array}{l}\text { Manimohan \& Leelavathy 1989d, Pradeep \& Vrinda 2010, } \\
\text { Mohanan } 2011 .\end{array}$ \\
\hline Inocybe ianthinofolia Pegler & Thi, Way, Pal, Way & Pradeep \& Vrinda 2010, Mohanan 2011. \\
\hline
\end{tabular}


Mycosphere Doi 10.5943/mycosphere/4/1/6

\begin{tabular}{|c|c|c|}
\hline Taxa & Distribution* & References \\
\hline Inocybe ingae Pegler & Thi, Ern & Pradeep \& Vrinda 2010, Mohanan 2011. \\
\hline Inocybe ionides Corner \& E. Horak & Pal & Vrinda et al. $1997 b$. \\
\hline Inocybe lasseri Dennis & Thi, Ern, Way & Vrinda et al. 1997b, Mohanan 2011. \\
\hline Inocybe littoralis Pegler & Ern & Pradeep et al. 1996a. \\
\hline Inocybe petchii Boedijn & Mal, Ern, Way, Thi & $\begin{array}{l}\text { Manimohan \& Leelavathy 1989d, Pradeep \& Vrinda 2010, } \\
\text { Mohanan } 2011 .\end{array}$ \\
\hline Inocybe purpureoflavida K.B. Vrinda \& C.K. Pradeep^${ }^{\wedge}$ & Thi, Ern, Kol & $\begin{array}{l}\text { Vrinda et al. 1997e, Pradeep \& Vrinda 2007, Pradeep \& } \\
\text { Vrinda 2010, Mohanan } 2011 .\end{array}$ \\
\hline Inocybe squamata J.E. Lange & Thi, Ern & Pradeep \& Vrinda 2010, Mohanan 2011. \\
\hline Inocybe stuntzii Grund & Idu, Thi & Vrinda et al. 2000b, Pradeep \& Vrinda 2010. \\
\hline Inocybe viridiumbonata Pegler & Way & Mohanan 2011. \\
\hline $\begin{array}{l}\text { Inocybe virosa K.B. Vrinda \& C.K. Pradeep, A.V. Joseph \& T.K. } \\
\text { Abraham^ }^{\wedge}\end{array}$ & $\begin{array}{l}\text { Thi, Pal, Ern, Thr, Idu, Kol, } \\
\text { Way }\end{array}$ & $\begin{array}{l}\text { Vrinda et al. 1996b, Pradeep \& Vrinda 2007, Pradeep \& } \\
\text { Vrinda 2010, Mohanan 2011, Pradeep \& Vrinda } 2011 .\end{array}$ \\
\hline Pleuroflammula flavomarginata (Berk. \& Broome) Singer & Ern & Mohanan 2011. \\
\hline Pleurotellus keralensis Sathe \& J.T. Daniel^ & Ern & Sathe \& Daniel 1980, Florence 2004. \\
\hline Tubaria virescens Noordel. \& K.B. Vrinda ${ }^{\wedge}$ & Thi & Noordeloos et al. 2007. \\
\hline \multicolumn{3}{|l|}{ Lyophyllaceae } \\
\hline Calocybe cyanocephala (Pat.) Pegler & Thr & Mohanan 2011. \\
\hline Lyophyllopsis keralensis Sathe \& J.T. Daniel^ & Idu & Sathe \& Daniel 1980, Florence 2004. \\
\hline Termitomyces clypeatus R. Heim & $\begin{array}{l}\text { Mal, Thr, Kol, Way, Thi, } \\
\text { Ern, Pal }\end{array}$ & $\begin{array}{l}\text { Leelavathy et al. 1983, Pradeep \& Vrinda 2007, Varghese et } \\
\text { al. 2010, Mohanan } 2011 .\end{array}$ \\
\hline Termitomyces entolomoides Heim & Mal, Thi & Leelavathy et al. 1983, Pradeep \& Vrinda 2007. \\
\hline Termitomyces eurrhizus (Berk.) R. Heim & $\begin{array}{l}\text { Kol, Ern, Thr, Way, Mal, } \\
\text { Thi }\end{array}$ & $\begin{array}{l}\text { Sathe \& Daniel } 1980 \text { (as T. quilonensis Sathe \& J.T. } \\
\text { Daniel^), Leelavathy et al. 1983, Sankaran \& Florence } \\
\text { 1995, Florence \& Yesodharan 1997, 2000, Florence 2004, } \\
\text { Pradeep \& Vrinda 2007, Varghese et al. 2010, Mohanan } \\
\text { 2011. }\end{array}$ \\
\hline Termitomyces globulus R. Heim \& Gooss.-Font & Mal, Kan & Leelavathy et al., 1983; Mohanan, 2011. \\
\hline Termitomyces heimii Natarajan & $\begin{array}{l}\text { Ern, Thr, Mal, Thi, Kol, } \\
\text { Way }\end{array}$ & $\begin{array}{l}\text { Sathe \& Daniel } 1980 \text { as Termitomyces longiradicata Sathe } \\
\text { \& Daniel\}, Leelavathy et al. 1983, Sankaran \& Florence } \\
\text { 1995, Florence \& Yesodharan 2000, Florence 2004, Pradeep } \\
\text { \& Vrinda 2007, Varghese et al. 2010. }\end{array}$ \\
\hline
\end{tabular}


Mycosphere Doi 10.5943/mycosphere/4/1/6

\begin{tabular}{|c|c|c|}
\hline Taxa & Distribution* & References \\
\hline Termitomyces le-testui (Pat.) R. Heim & Way & Mohanan 2011. \\
\hline Termitomyces mammiformis R. Heim & Mal, Thi, Ern & $\begin{array}{l}\text { Leelavathy et al. 1983, Bhavanidevi \& Nair 1983, Florence } \\
\text { 2004, Mohanan } 2011 .\end{array}$ \\
\hline Termitomyces microcarpus (Berk. \& Broome) R. Heim & $\begin{array}{l}\text { Ern, Thr, Mal, Idu, Thi, Kol, } \\
\text { Way }\end{array}$ & $\begin{array}{l}\text { Sathe \& Daniel } 1980 \text { \{as Podabrella microcarpa (Berk. \& } \\
\text { Broome) Singer\}, Leelavathy et al. 1983, Sankaran \& } \\
\text { Florence 1995, Florence \& Yesodharan 1997, 2000, } \\
\text { Mohanan 2003, Florence 2004, Pradeep \& Vrinda 2007, } \\
\text { Varghese et al. 2010, Mohanan 2011. }\end{array}$ \\
\hline Termitomyces perforans Heim & Mal & Leelavathy et al. 1983. \\
\hline Termitomyces robustus (Beeli) R. Heim & Thi, Kan & $\begin{array}{l}\text { Bhavanidevi et al . 1980, Bilgrami et al. 1991, Florence } \\
\text { 2004, Mohanan } 2011 .\end{array}$ \\
\hline Termitomyces sagittiformis (Kalchbr. \& Cooke) D.A. Reid & Thi & Vrinda \& Pradeep 2009 \\
\hline Termitomyces schimperi (Pat.) R. Heim & Mal, Thr & Mohanan 2011. \\
\hline Termitomyces striatus (Beeli) R. Heim & Mal, Way & Leelavathy et al. 1983, Mohanan 2011. \\
\hline Termitomyces tylerianus Otieno & Mal & Mohanan 2011. \\
\hline Termitomyces umkowaan (Cooke \& Mass.) D.A. Reid & Thi, Thr & $\begin{array}{l}\text { Vrinda et al. 2002a }\{\text { as } T \text {. umkowaani }\} \text {, Pradeep \& Vrinda } \\
2007 \text { as T. umkowaani }\}, \text { Mohanan } 2011 .\end{array}$ \\
\hline \multicolumn{3}{|l|}{ Marasmiaceae } \\
\hline Calyptella capula (Holmsk.) Quél. & $\mathrm{Pal}$ & Mohanan 2011. \\
\hline Campanella pustulata (Berk. \& Broome) Lloyd & Thr & Mohanan 2011. \\
\hline Chaetocalathus niduliformis (Murrill) Singer & Thi & Vrinda et al. 2000a. \\
\hline Chaetocalathus semisupinus (Berk. \& Broome) Pegler & Mal & Manimohan \& Leelavathy 1989b. \\
\hline Gerronema tenue Dennis & Ern & Mohanan 2011. \\
\hline Gymnopus androsaceus (L.) J.L. Mata \& R.H. Petersen & Thr & $\begin{array}{l}\text { Florence \& Yesodharan } 2000 \text {, Florence } 2004 \text { \{as } \\
\text { Marasmius androsaceus (L.) Fr. }\} .\end{array}$ \\
\hline Gymnopus dryophilus (Bull.) Murrill & Way & Mohanan 2011 \\
\hline Hydropus anthidepas (Berk. \& Broome) Singer & Thi & Pradeep et al. 1996a. \\
\hline Hydropus cylindrocystis Pegler & Thr & Mohanan 2011. \\
\hline Hydropus sphaerosporus (Dennis) Dennis & Thi & Mohanan 2011. \\
\hline Lactocollybia epia (Berk. \& Broome) Pegler & Way & Mohanan, 2011. \\
\hline Lactocollybia ianthina R. Heim ex Singer & Idu & Sathe \& Daniel 1980, Florence 2004. \\
\hline
\end{tabular}


Mycosphere Doi 10.5943/mycosphere/4/1/6

\begin{tabular}{|c|c|c|}
\hline Taxa & Distribution* & References \\
\hline Marasmiellus echinocephalus Singer & Thi & Vrinda et al. 2000b. \\
\hline Marasmiellus fusicystis Manim. \& Leelav. ^ & Way & Manimohan \& Leelavathy 1988e. \\
\hline Marasmiellus ignobilis (Berk. \& Broome) Pegler & Mal & Sankaran \& Florence 1993, Florence 2004, Mohanan 2011. \\
\hline Marasmiellus inoderma (Berk.) Singer & Koz & $\begin{array}{l}\text { Nag Raj } 1965 \text { \{as } M \text {. indoderma (Berk.) Singer\}, Bilgrami } \\
\text { et al. } 1991 \text {, Florence } 2004 .\end{array}$ \\
\hline Marasmiellus keralensis Sathe \& J.T. Daniel^ & Thi & $\begin{array}{l}\text { Sathe \& Daniel } 1980 \text { \{as Marasmiellus keralense Sathe } \\
\text { \&Daniel\}, Florence } 2004 .\end{array}$ \\
\hline Marasmiellus scandens (Massee) Dennis \& D. A. Reid & Kot & $\begin{array}{l}\text { Chandramohanan \& Kaveriappa 1983, Bilgrami et al. 1991, } \\
\text { Florence } 2004 \text { (as Marasmius scandens Massee). }\end{array}$ \\
\hline Marasmiellus stenophyllus (Mont.) Singer & Thr & Mohanan 2011. \\
\hline Marasmiellus subaurantiacus (Berk. \& Broome) Pegler & Thr & Mohanan 2011. \\
\hline Marasmius amygdalosporus Manim. \& Leelav. ^ & Way & Manimohan \& Leelavathy 1988a. \\
\hline Marasmius atrorubens (Berk.) Sacc. & Way, Mal, Thr & $\begin{array}{l}\text { Manimohan \& Leelavathy } 1989 \text { a }\{\text { as } M . \text { atrorubens var. } \\
\text { atrorubens (Berk.) Berk. and M. atrorubens var. dumontii } \\
\text { Singer\}, Mohanan } 2011 .\end{array}$ \\
\hline Marasmius brunneolus (Berk. \& Br.) Pegler & Way & Manimohan \& Leelavathy 1989a. \\
\hline Marasmius caryotae (Berk.) Petch & Thi & Joseph et al. 1995. \\
\hline Marasmius confertus Berk.\& Broome & Thr & Mohanan 2011. \\
\hline Marasmius coniatus Berk. \& Broome & Way & Manimohan \& Leelavathy 1989a. \\
\hline Marasmius corrugatiformis Singer & Mal, Thr & Mohanan 2011. \\
\hline Marasmius elaeocephalus Singer & Idu & Mohanan 2011. \\
\hline Marasmius epiphyllus (Pers.) Fr. & Ern & Florence 2004. \\
\hline Marasmius flexipes Manim. \& Leelav. ^ & Mal & Manimohan \& Leelavathy $1987 b$. \\
\hline Marasmius florideus Berk. \& Broome & Mal & Manimohan \& Leelavathy 1989a. \\
\hline Marasmius fulviceps Berk. & Way & Manimohan \& Leelavathy 1989a. \\
\hline Marasmius gordipes Sacc. \& Paol. & Way & Manimohan \& Leelavathy 1989a. \\
\hline Marasmius grandisetulosus Singer & Idu & $\begin{array}{l}\text { Natarajan \& Manjula, 1982, Bilgrami et al. 1991, Florence } \\
2004 .\end{array}$ \\
\hline Marasmius griseoroseus (Mont.) Dennis & Mal & Manimohan \& Leelavathy 1989a. \\
\hline Marasmius haematocephalus (Mont.) Fr. & Ern, Thr, Mal & $\begin{array}{l}\text { Sankaran \& Florence 1995, Florence \& Yesodharan 1997, } \\
\text { 2000, Florence 2004, Mohanan } 2011 .\end{array}$ \\
\hline Marasmius hakgalensis Petch & Way, Thi & Mohanan, 2011. \\
\hline
\end{tabular}


Mycosphere Doi 10.5943/mycosphere/4/1/6

\begin{tabular}{|c|c|c|}
\hline Taxa & Distribution* & References \\
\hline Marasmius hypochroides Berk. \& Broome & Mal & Manimohan \& Leelavathy 1989a, Mohanan 2011. \\
\hline Marasmius kisangensis Singer & Thr & Mohanan 2011. \\
\hline Marasmius leelavathyi Manim., Tkalčec \& Mešić^ & Mal & $\begin{array}{l}\text { Manimohan \& Leelavathy 1987a (as Marasmius parvulus } \\
\text { Manim. \& Leelav.^), Mešić \& Tkalčec } 2010 .\end{array}$ \\
\hline Marasmius leveilleanus (Berk.) Sacc. & Way & Mohanan 2011. \\
\hline Marasmius nigrobrunneus (Pat.) Sacc. & Way & Manimohan \& Leelavathy 1989a. \\
\hline Marasmius purpureoalbus Petch & Thr & Florence \& Yesodharan 1997, 2000, Florence 2004. \\
\hline Marasmius rigidichorda Petch & Mal & Mohanan 2011. \\
\hline Marasmius spegazzinii (Kuntze) Sacc. \& P. Syd. & Ern & Mohanan 2011. \\
\hline Marasmius subconiatus Petch & Mal & Manimohan \& Leelavathy 1989a. \\
\hline Marasmius tubulatus Petch & Mal & Manimohan \& Leelavathy 1989a. \\
\hline Megacollybia platyphylla (Pers.) Kotl. \& Pouzar & Idu & Mohanan 2011. \\
\hline Megacollybia virosa Manim. \& K.B. Vrinda ${ }^{\wedge}$ & Koz, Thi & Manimohan et al. 2010. \\
\hline Micromphale orientale Manim. \& Leelav. ^ & Way & Manimohan \& Leelavathy 1988a. \\
\hline Nothopanus eugrammus (Mont.) Singer & Thi, Kol & $\begin{array}{l}\text { Vrinda et al. 1999a, Pradeep \& Vrinda 2007, Pradeep \& } \\
\text { Vrinda 2011. }\end{array}$ \\
\hline Nothopanus minutus Sathe \& J.T. Daniel^ & Idu & Sathe \& Daniel 1980, Florence 2004 \\
\hline Omphalotus japonicas (Kawam.) Kirchm. \& O.K. Mill. & Thr & $\begin{array}{l}\text { Sathe \& Daniel 1980, Florence } 2004 \text { \{as Lampteromyces } \\
\text { japonicus (Kawam.) Singer\}. }\end{array}$ \\
\hline Omphalotus olearius (DC.) Singer & Thi, Ern, Ala, Way & $\begin{array}{l}\text { Vrinda et al. 1999a, Pradeep \& Vrinda 2007, Mohanan, } \\
\text { 2011, Pradeep \& Vrinda } 2011 .\end{array}$ \\
\hline Tetrapyrgos nigripes (Fr.) E. Horak & Thr & Mohanan 2011. \\
\hline Tetrapyrgos simulans (Pat.) E. Horak & Way & $\begin{array}{l}\text { Manimohan \& Leelavathy 1988a (as Campanella simulans } \\
\text { var. bispora Manim. \& Leelav. ^^). }\end{array}$ \\
\hline Trogia cantharelloides (Mont.) Pat. & Thi & Mohanan 2011. \\
\hline Trogia cyanea Corner & Koz, Way & Kumar \& Manimohan 2009d. \\
\hline Trogia infundibuliformis Berk. \& Broome & $\mathrm{Koz}$ & Kumar \& Manimohan 2009d, Mohanan 2011. \\
\hline \multicolumn{3}{|l|}{ Mycenaceae } \\
\hline Favolaschia thwaitesii (Berk. \& Broome) Kuntze & Way & Mohanan 2011. \\
\hline Favolaschia tonkinensis (Pat.) Kuntze & Way, Thr & Pradeep et al. 2008, Mohanan 2011. \\
\hline
\end{tabular}


Mycosphere Doi 10.5943/mycosphere/4/1/6

\begin{tabular}{|c|c|c|}
\hline Taxa & Distribution* & References \\
\hline Filoboletus manipularis (Berk.) Singer & Way, Thr, Idu, Ern, Pal & Manimohan \& Leelavathy 1989b, Mohanan 2011. \\
\hline Hemimycena longicystis R. Heim & Thi & Vrinda et al., 1996c. \\
\hline Mycena alphitophora (Berk.) Sacc. & Mal, Pal, Thr & 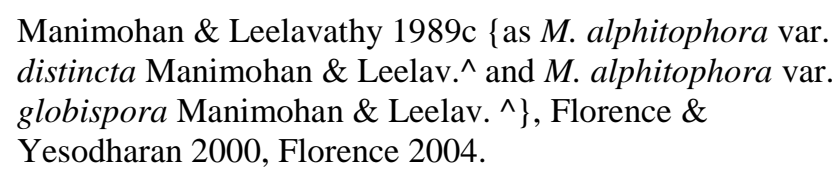 \\
\hline Mycena auroricolor (Berk. \& Broome) Petch & Mal, Way & Manimohan et al. 1988, Mohanan 2011. \\
\hline Mycena haematopus (Pers.) P. Kumm & Thi & Bhavanidevi \& Nair 1983, Florence 2004. \\
\hline Mycena indica Manim. \& Leelav. ^ & Mal & Manimohan \& Leelavathy 1988c. \\
\hline Mycena profusa Manim. \& Leelav. ^ & Mal & Manimohan \& Leelavathy 1988c. \\
\hline Mycena pura (Pers.) P. Kumm. & Ern & Mohanan 2011. \\
\hline Xeromphalina tenuipes (Schwein.) A.H. Sm. & Thr & Mohanan 2011. \\
\hline \multicolumn{3}{|l|}{ Physalacreaceae } \\
\hline Armillaria mellea (Vahl) P. Kumm. & Idu & Mohanan 2011. \\
\hline Cyptotrama asprata (Berk.) Redhead \& Ginns. & Idu, Mal, Way & Mohanan 2011. \\
\hline Cyptotrama lachnocephala(Pat.) Singer & Kan, Mal, Way & Mohanan 2011 \{as C. lachnocephalum (Pat.) Singer\}. \\
\hline Gloiocephala resinopunctata Manim. \& K.A. Thomas^ & Kan, Mal & Manimohan \& Thomas 1998, Mohanan 2011. \\
\hline Oudemansiella canarii (Jungh.) Höhn. & Way, Pal, Mal & Varghese et al. 2010, Mohanan 2011. \\
\hline Oudemansiella munnarensis Sathe \& J.T. Daniel^ & Idu & Sathe \& Daniel 1980, Florence 2004. \\
\hline Oudemansiella radicata (Rehl. Ex Fr.) Singer & Way & Varghese et al. 2010. \\
\hline Xerula furfuracea (Peck) Redhead, Ginns \& Shoemaker & Thr, Idu & Mohanan 2011. \\
\hline Xerula megalospora (Clem.) Redhead, Ginns \& Shoemaker & Pal, Mal & Mohanan 2011. \\
\hline Xerula radicata (Relhan) Dörfelt & Thr, Kol & Mohanan 2011. \\
\hline $\begin{array}{l}\text { Xerula rubrobrunnescens Redhead, Ginns \& Shoemaker } \\
\text { Pleurotaceae }\end{array}$ & Pal & Mohanan 2011. \\
\hline Hohenbuehelia aurantiocystis Pegler & Ern & Mohanan 2011. \\
\hline Hohenbuehelia pergelatinosa Singer & Thi & Vrinda et al. 1996c. \\
\hline Hohenbuehelia petaloides (Bull.) Schulzer & Ern & Mohanan 2011. \\
\hline Hohenbuehelia testudo (Berk.) Pegler & Pal, Ern & Mohanan 2011. \\
\hline
\end{tabular}


Mycosphere Doi 10.5943/mycosphere/4/1/6

\begin{tabular}{|c|c|c|}
\hline Taxa & Distribution* & References \\
\hline Pleurotus citrinopileatus Singer & Thi, Idu & $\begin{array}{l}\text { Justin \& Abraham 1988, Geetha \& Sivaprakasham 1998, } \\
\text { Florence 2004, Mohanan } 2011 .\end{array}$ \\
\hline Pleurotus djamor (Rumph. ex Fr.) Boedijn & Thi, Way, Thi, Thr & $\begin{array}{l}\text { Geetha \& Sivaprakasham 1998, Ouseph et al. 2001, } \\
\text { Florence 2004, Mohanan } 2011 .\end{array}$ \\
\hline Pleurotus eöus (Berk.) Sacc. & Thi, Kol, Thr, Ern, way & Varghese et al., 2010; Mohanan, 2011. \\
\hline Pleurotus flabellatus Sacc. & Thi, Way, Thr, Ern, Mal & $\begin{array}{l}\text { Abraham \& Pradeep 1995, Florence 2004, Varghese et al. } \\
\text { 2010, Mohanan } 2011 .\end{array}$ \\
\hline IIPleurotus florida (Berk.) Sacc. & Thr & Florence \& Balasundaran 2000, Florence 2004. \\
\hline Pleurotus ostreatus (Jacq.) P.Kumm. & Way & Mohanan 2011. \\
\hline Pleurotus platypus Sacc. & Thi & Ouseph et al. 2001, Florence 2004. \\
\hline \multicolumn{3}{|l|}{ Pluteaceae } \\
\hline Pluteus aeolus Berk. \& Broome & Way & Mohanan 2011. \\
\hline Pluteus aglaeotheles Berk. \& Broome & Mal & Mohanan 2011. \\
\hline Pluteus amphicystis Singer & & Pradeep et al. 2002. \\
\hline Pluteus atromarginatus (Konard) Kühner & Thr & Mohanan 2011. \\
\hline Pluteus cervinus (Schaeff.) P. Kumm. & Idu & Pradeep et al. 2002. \\
\hline Pluteus chrysaegis (Berk. \& Broome) Petch & Kan & Pradeep \& Vrinda 2006. \\
\hline Pluteus conizatus (Berk. \& Broome) Sacc. & Thi, Kol & Mohanan 2011. \\
\hline Pluteus cubensis (Murrill) Dennis & Thi & Pradeep \& Vrinda 2005b. \\
\hline Pluteus delicatulus C.K. Pradeep \& K.B. Vrinda^ & Thi & Pradeep \& Vrinda 2006. \\
\hline Pluteus escharites (Berk. \& Broome) Sacc. & Way & Mohanan 2011. \\
\hline Pluteus eupigmentatus Singer & Thi & Pradeep \& Vrinda 2005b. \\
\hline Pluteus fastigiatus Singer & Thi, Mal & Pradeep \& Vrinda 2005b, Mohanan 2011. \\
\hline Pluteus fusconigricans (Berk. \& Broome) Sacc. & Thi, Mal & Pradeep \& Vrinda 2005a, Mohanan 2011. \\
\hline Pluteus glyphidiatus (Berk. \& Broome) Sacc. & Thr & Mohanan 2011. \\
\hline Pluteus haywardii Singer & Thi, Pal, Thr & Pradeep \& Vrinda 2005b, Mohanan 2011. \\
\hline Pluteus luteostipitatus C.K. Pradeep \& K.B. Vrinda^ & Thi & Pradeep \& Vrinda 2008. \\
\hline Pluteus martinicensis Singer \& Fiard & Thi & Pradeep et al. 1996a, 2002. \\
\hline Pluteus pelinus (Berk. \& Broome) Sacc. & Mal & Mohanan 2011. \\
\hline
\end{tabular}


Mycosphere Doi 10.5943/mycosphere/4/1/6

\begin{tabular}{|c|c|c|}
\hline Taxa & Distribution* & References \\
\hline Pluteus podospileus Sacc. \& Cub. & Koz, Ern & Pradeep \& Vrinda 2005a, Mohanan 2011. \\
\hline Pluteus pulverulentus Murrill & Thi, Pal & Pradeep \& Vrinda 2005a, Mohanan 2011. \\
\hline Pluteus silentvalleyanus C.K. Pradeep \& K.B. Vrinda^ & Pal & Pradeep \& Vrinda 2008 \{as P. silentvalliyanus $\}.$ \\
\hline Pluteus subcervinus (Berk. \& Broome) Sacc. & Pal, , Idu & Pradeep et al. 2002, Mohanan 2011. \\
\hline Volvariella apalotricha (Berk. \& Broome) Pegler & Thi & Pradeep et al. 1998. \\
\hline Volvariella bombycina (Schaeff.) Singer & Ern & Pradeep et al., 1998. \\
\hline Volvariella cubensis (Murrill) Shaffer & Thr & Mohanan 2011. \\
\hline Volvariella glandiformis (Berk. \& Broome) Pegler & Thi, Thr & Pradeep et al. 1998, Mohanan 2011. \\
\hline Volvariella hypopithys (Fr.)Shaffer & Pal, Thi & Pradeep et al. 1998. \\
\hline Volvariella nigrodisca Shaffer & Thi, Ern & Pradeep et al. 1998, Mohanan 2011. \\
\hline Volvariella pseudovolvacea (Berk. \& Broome) Singer & Thi, Ern, Mal & Pradeep et al. 1998, Mohanan 2011. \\
\hline Volvariella pusilla (Pers.) Singer & Thi & Pradeep et al. 1998. \\
\hline Volvariella taylorii (Berk. \& Broome) Singer & Pal, Thr & $\begin{array}{l}\text { Pradeep et al. } 1998 \text { \{as V. taylori (Berk.) Singer\}, Mohanan } \\
\text { 2011. }\end{array}$ \\
\hline Volvariella terastia (Berk. \& Broome) Singer & $\mathrm{Koz}$ & Manimohan et al. 1988. \\
\hline Volvariella volvacea (Bull.) Singer & Thi, Way, Mal, Thr, Kol & $\begin{array}{l}\text { Lakshmanan et al. 1979, Pradeep et al. 1998, Florence 2004, } \\
\text { Manimohan et al. 2007, Pradeep \& Vrinda 2007, Mohanan } \\
2011 .\end{array}$ \\
\hline Volvopluteus gloiocephalus (DC.) Justo & Idu, Thr & $\begin{array}{l}\text { Pradeep et al. } 1998 \text { \{as Volvariella speciosa (Fr.) Singer\}, } \\
\text { Mohanan } 2011 \text { as Volvariella gloiocephala (DC.) } \\
\text { Boekhout \& Enderle\}. }\end{array}$ \\
\hline \multicolumn{3}{|l|}{ Psathyrellaceae } \\
\hline Coprinellus disseminatus (Pers.) J.E. Lange & Mal, Thr & Mohanan 2011 as Coprinus disseminatus (Pers.) Gray\}. \\
\hline $\begin{array}{l}\text { Coprinopsis fibrillosa (Berk. \& Broome) Redhead,Vilgalys \& } \\
\text { Moncalvo }\end{array}$ & Thr, Mal & Mohanan 2011. \\
\hline Cystoagaricus trisulphuratus (Berk.) Singer & Way, Mal, Thi, Ala, Pal, Kol & $\begin{array}{l}\text { Pradeep \& Vrinda } 2007 \text { (as Agaricus trisulphuratus Berk.), } \\
\text { Mohanan } 2011 .\end{array}$ \\
\hline Lacrymaria lacrymabunda (Bull.) Pat. & Idu & Vrinda et al. 2003a \{as Psathyrella velutina (Pers.) Singer $\}.$ \\
\hline Parasola plicatilis (Curtis) Redhead,Vilgalys \& Hopple & Idu, Way, Thr & $\begin{array}{l}\text { Natarajan \& Raman 1983, Florence } 2004\{\text { as Coprinus } \\
\text { plicatilis var. microsporus Kühner \& Joss.\}, Mohanan } \\
2011 \text { as Coprinus plicatilis var. plicatilis (Curtis) Fr.\}. }\end{array}$ \\
\hline
\end{tabular}


Mycosphere Doi 10.5943/mycosphere/4/1/6

\begin{tabular}{|c|c|c|}
\hline Taxa & Distribution* & References \\
\hline Psathyrella candolleana (Fr.) Maire & Mal, Idu, Thr & Mohanan 2011. \\
\hline${ }^{\mathbb{1}}$ Psathyrella conopilea (Fr.) Pearson \& Dennis & Thi & Vrinda et al. 1999b. \\
\hline Psathyrella coprinoceps (Berk. \& M.A. Curtis) Dennis & Thi & Vrinda et al. 2001. \\
\hline Psathyrella efflorescens (Sacc.) Pegler & Ern & Mohanan 2011. \\
\hline Psathyrella lithocarpi A.H. Sm. & Way & Mohanan 2011. \\
\hline Psathyrella lucipeta (Berk. \& Broome) Pegler & Ern & Mohanan 2011. \\
\hline Psathyrella myceniformis Dennis & $\mathrm{Pal}$ & Mohanan 2011. \\
\hline Psathyrella trechispora (Petch) Pegler & Ern, Thi, Mal & Pradeep et al., 1996a; Mohanan, 2011. \\
\hline \multicolumn{3}{|l|}{ Strophariaceae } \\
\hline Agrocybe calicutensis K.A. Thomas \& Manim. ^ & Mal & Thomas \& Manimohan 2003. \\
\hline Agrocybe guruvayoorensis K.A. Thomas \& Manim. ^ & Thr & Thomas \& Manimohan 2003, Manimohan et al. 2007. \\
\hline Agrocybe manihotis Pegler & Idu & $\begin{array}{l}\text { Natarajan \& Raman 1983, Florence } 2004 \text { (as Agrocybe } \\
\text { manihotis var. microspora Natarajan \& } \operatorname{Raman}^{\wedge} \text { ). }\end{array}$ \\
\hline Agrocybe munnarensis K.A. Thomas \& Manim. ${ }^{\wedge}$ & Idu & Thomas \& Manimohan 2003. \\
\hline Agrocybe pediades (Fr.) Fayod & Way, Mal, Idu & $\begin{array}{l}\text { Thomas \& Manimohan } 2003 \text { \{as Agrocybe temulenta (Fr.) } \\
\text { Singer.\}, Mohanan } 2011 .\end{array}$ \\
\hline Agrocybe retigera (Speg.) Singer & Mal & Mohanan 2011. \\
\hline Agrocybe wayanadensis K.A. Thomas \& Manim. ${ }^{\wedge}$ & Way, Thr & Thomas \& Manimohan 2003, Mohanan 2011. \\
\hline Galerina vittiformis (Fr.) Singer & Kan & Mohanan 2011. \\
\hline Gymnopilus bryophilus Murrill & Way, Mal & Thomas et al. 2003, Mohanan 2011. \\
\hline Gymnopilus chrysopellus (Berk. \& M.A. Curtis) Murrill & Mal & Mohanan 2011. \\
\hline Gymnopilus crocias (Berk. \& Broome) Singer & Mal & Mohanan 2011. \\
\hline Gymnopilus dilepis (Berk. \& Broome) Singer & Mal, Way & Thomas et al. 2003, Mohanan 2011. \\
\hline Gymnopilus hispidellus Murrill & $\mathrm{Pal}$ & Mohanan 2011. \\
\hline Gymnopilus junonius (Fr.) P.D. Orton & Thr, Way & Mohanan 2011. \\
\hline Gymnopilus lateritius (Pat.) Murrill & Idu & Mohanan 2011. \\
\hline Gymnopilus minutosporus Natarajan \& Raman^ & Idu & Natarajan \& Raman 1983a, Florence 2004. \\
\hline Gymnopilus subbellulus Hesler & Pal & Mohanan 2011. \\
\hline
\end{tabular}


Mycosphere Doi 10.5943/mycosphere/4/1/6

\begin{tabular}{|c|c|c|c|}
\hline Taxa & & Distribution* & References \\
\hline & Gymnopilus terricola K.A. Thomas, Guzm.-Dav. \& Manim. ^ & Mal, Thr & Thomas et al. 2003, Mohanan 2011. \\
\hline & Gymnopilus zenkeri (Henn.) Singer & Thr & Vrinda et al. 1997d, Mohanan 2011. \\
\hline & Hypholoma subviride (Berk. \& M.A. Curtis) Dennis & Ern & Mohanan 2011. \\
\hline & Hypholoma trinitense (Dennis) Pegler & Thi, Ern & Vrinda et al. 1996c \{as H. trinitensis\}, Mohanan 2011. \\
\hline & Melanotus subcuneiformis (Murrill) Singer & Kot & Sathe \& Daniel 1980, Florence 2004. \\
\hline & Naematoloma munnarense Sathe \& J.T. Daniel^ & Idu & $\begin{array}{l}\text { Sathe \& Daniel } 1980 \text { as Naematoloma munnarensis Sathe } \\
\text { \& Daniel\}, Florence } 2004 .\end{array}$ \\
\hline & Naucoria escharioides (Fr.) P. Kumm. & Ern & Florence 2004. \\
\hline & Pholiota bambusina K.A. Thomas \& Manim.^ & Way & Thomas \& Manimohan 2001. \\
\hline & Pholiota ealaensis Beeli & Thi & Joseph et al. 1995. \\
\hline & Pholiota gregariiformis (Mirrill) A.H. Sm. \& Hesler & Idu & Mohanan, 2011. \\
\hline & Pholiota squarrosa (Vahl) P. Kumm. & Thr & Mohanan 2011. \\
\hline & Psilocybe argentina (Speg.) Singer & Idu, Mal & Thomas et al. 2002b, Mohanan 2011. \\
\hline & Psilocybe aztecorum R. Heim & Thr, Idu, Thi & Mohanan 2011. \\
\hline & Psilocybe coprophila (Bull.) P. Kumm. & Idu & Thomas et al. 2002b, Manimohan et al. 2007. \\
\hline & Psilocybe cubensis (Earle) Singer & Idu, Way, Pal, Thi & $\begin{array}{l}\text { Thomas et al. 2002b, Pradeep \& Vrinda 2007, Mohanan } \\
\text { 2011, Pradeep \& Vrinda } 2011 .\end{array}$ \\
\hline & Psilocybe goniospora (Berk. \& Broome) Singer & Ern & Vrinda et al. 2001 \\
\hline & Psilocybe indica Sathe \& J.T. Daniel^ & Idu & Sathe \& Daniel 1980, Florence 2004. \\
\hline & Psilocybe inquilina (Fr.) Bres. & Mal & Mohanan 2011. \\
\hline & Psilocybe keralensis K.A. Thomas, Manim. \& Guzmán^ & Way & Thomas et al. $2002 \mathrm{~b}$. \\
\hline & Psilocybe pegleriana Guzmán & Thr, Pal, Kas & Thomas et al. 2002b, Manimohan et al. 2007. \\
\hline & Psilocybe plutonia (Berk. \& M.A. Curtis) Sacc. & Ern, Mal & Mohanan 2011. \\
\hline & Psilocybe samuiensis Guzmán, Bandala \& J.W. Allen & Idu & Mohanan 2011. \\
\hline & Psilocybe squarrosipes Singer & Idu & Mohanan 2011. \\
\hline & Psilocybe subaeruginascens Höhn. & Koz & Thomas et al. 2002b, Manimohan et al. 2007. \\
\hline & Psilocybe subcubensis Guzmán & Koz, Way, Mal, Kol & $\begin{array}{l}\text { Thomas et al. 2002b, Manimohan et al. 2007, Mohanan } \\
\text { 2011, Pradeep \& Vrinda } 2011 .\end{array}$ \\
\hline & Psilocybe wayanadensis K.A. Thomas, Manim. \& Guzmán^ & Way, Ern & Thomas et al. 2002b, Mohanan 2011. \\
\hline & Stropharia bicolor Pegler & Idu, Pal & Manimohan et al. 2007. \\
\hline
\end{tabular}


Mycosphere Doi 10.5943/mycosphere/4/1/6

\begin{tabular}{|c|c|c|}
\hline Taxa & Distribution* & References \\
\hline Stropharia rugosoannulata Farl. ex Murrill & Way, Pal & Manimohan et al. 2007. \\
\hline Stropharia semiglobata (Batsch) Quél & Mal, Way & Mohanan 2011, Pradeep \& Vrinda 2011. \\
\hline \multicolumn{3}{|l|}{ Tricholomataceae } \\
\hline Amparoina spinosissima (Singer) Singer & Koz & Aravindakshan \& Manimohan 2010. \\
\hline Callistosporium luteo-olivaceum (Berk. \& M.A. Curtis) Singer & Way & Manimohan \& Leelavathy 1989b. \\
\hline Clitocybe mayyanadensis Sathe \& J.T. Daniel^ ${ }^{\wedge}$ & Kol & Sathe \& Daniel 1980, Florence 2004. \\
\hline Clitocybe rivulosa (Pers.) P. Kumm. & Way & Mohanan 2011 as Clitocybe dealbata (Sowerby) Gillet\}. \\
\hline Collybia aurea (Beeli) Pegler & Thi & Mohanan 2011. \\
\hline Collybia bakeri Dennis & Mal & Mohanan 2011. \\
\hline Collybia chrysoropha (Berk. \& Broome) Sacc. & Mal & Mohanan 2011. \\
\hline Collybia coracicolor (Berk. \& M. A. Curtis) Dennis & Way & Mohanan, 2011. \\
\hline Collybia leucophaea Sacc. & Ern, Thr, Way & $\begin{array}{l}\text { Sankaran 1993, Sankaran \& Florence 1995, Florence \& } \\
\text { Yesodharan 1997, 2000, Florence 2004, Mohanan } 2011 .\end{array}$ \\
\hline Collybia multijuga (Berk. \& Broome) Sacc. & Mal & Mohanan 2011. \\
\hline Collybia purpureogrisea (Petch) Pegler & Pal, Ern & $\begin{array}{l}\text { Manimohan \& Leelavathy 1989b, Sankaran \& Florence } \\
\text { 1995, Florence } 2004 \text { as Marasmiellus purpureoalbus } \\
\text { (Petch) Singer }\}\end{array}$ \\
\hline Collybia rufipicta (Berk. \& Broome) Sacc. & Thr & Mohanan 2011. \\
\hline Collybia sublaccata (Berk. \& Broome) Pegler & Kol & Mohanan 2011. \\
\hline Collybia syringea Singer & Mal & Mohanan 2011. \\
\hline Dermoloma cystidiatum Manim. \& Arnolds & Mal & Manimohan \& Arnolds 1998. \\
\hline Lepista hyalodes (Berk. \& Broome) Pegler & Mal, Ern, Thr & $\begin{array}{l}\text { Manimohan \& Leelavathy 1989b, Sankaran \& Florence } \\
\text { 1995, Florence 2004, Mohanan } 2011 .\end{array}$ \\
\hline Lepista indica Sathe \& J.T. Daniel ${ }^{\wedge}$ & Idu & Sathe \& Daniel 1980, Florence 2004. \\
\hline Lepista sordida (Schumach.) Singer & Mal, Thr, Way & Varghese et al. 2010, Mohanan 2011. \\
\hline Macrocybe gigantea (Massee) Pegler \& Lodge & Thr & Manimohan et al. 2007. \\
\hline Macrocybe lobayensis (R. Heim) Pegler \& Lodge & Thi, Kol, Ern & $\begin{array}{l}\text { Balakrishnan \& Nair 1998, Vrinda \& Pradeep 2006, Pradeep } \\
\text { \& Vrinda } 2007 \text { \{as Macrocybe lobayense\}, Mohanan } 2011 .\end{array}$ \\
\hline Macrocybe pachymeres (Berk. \& Broome) Pegler \& Lodge & Thr & Mohanan 2011. \\
\hline Macrocybe titans (H.E. Bigelow \& Kimbr.) Pegler, Lodge \& Nakasone & Thi & $\begin{array}{l}\text { Vrinda et al. 1997f (as Tricholoma titans H. E. Bigelow \& } \\
\text { Kimbr.). }\end{array}$ \\
\hline Mniopetalum minutum Manim. \& Leelav. ^ & Mal & Manimohan \& Leelavathy 1988d. \\
\hline Phaeomycena indica Sathe \& J.T. Daniel^ & Kot & Sathe \& Daniel 1980, Florence 2004. \\
\hline Pleuromycenula hydrophila Manim. \& Leelav. ^^ & Mal & Manimohan \& Leelavathy 1987c. \\
\hline Pseudobaeospora lavendulamellata Arnolds, Leelav. \& Manim.^ & Mal & Arnolds et al. 2004. \\
\hline
\end{tabular}


Mycosphere Doi 10.5943/mycosphere/4/1/6

\begin{tabular}{|c|c|c|}
\hline Taxa & Distribution* & References \\
\hline Tricholoma ceriniceps Pegler & Mal & Mohanan 2011. \\
\hline Tricholoma magnivelare (Peck) Redhead & Mal & Mohanan 2011 (as Agaricus magnivelaris Peck). \\
\hline Tricholoma rhacophorum (Berk. \& Broome) Sacc. & Mal & Manimohan \& Leelavathy 1989 b. \\
\hline Tricholoma rimosoides Dennis & Thr & Mohanan 2011. \\
\hline Tricholoma subrimosum (Murrill) Murrill & Way & Mohanan 2011. \\
\hline \multicolumn{3}{|l|}{ Incertae sedis } \\
\hline Coniolepiota spongodes (Berk. \& Broome) Vellinga & Way & $\begin{array}{l}\text { Mohanan } 2011 \text { as Lepiota spongodes (Berk. \& Broome) } \\
\text { Sacc.\}. }\end{array}$ \\
\hline Copelandia tropica Natarajan \& Raman^ & Idu & Natarajan \& Raman 1983, Florence 2004. \\
\hline Panaeolina foenisecii (Pers.) Maire & Idu & Mohanan 2011. \\
\hline Panaeolina indica Sathe \& J.T. Daniel^ & Idu & Sathe \& Daniel 1980, Florence 2004. \\
\hline Panaeolus acuminatus (Schaeff.) Quél. & Pal, Thi, Thr, Mal, Ern, Way & $\begin{array}{l}\text { Vrinda et al. 1999b, Manimohan et al. } 2007 \text { (as } P \text {. rickenii } \\
\text { Hora), Mohanan 2011 \{as P. acuminatus var. rickenii (Hora) } \\
\text { P. Roux, Guy García \& Charret\}. }\end{array}$ \\
\hline Panaeolus africanus Ola'h & Thi, Kol & Pradeep \& Vrinda, 2011. \\
\hline Panaeolus antillarum (Fr.) Dennis & $\begin{array}{l}\text { Mal, Thr, Way, Thi, Idu, } \\
\text { Pal, Ern, Kol }\end{array}$ & $\begin{array}{l}\text { Manimohan et al. 2007, Mohanan 2011, Pradeep \& Vrinda } \\
2011 .\end{array}$ \\
\hline Panaeolus cyanescens (Berk. \& Broome) Sacc. & Mal, Way, Thr, Thi & $\begin{array}{l}\text { Manimohan et al. 2007, Mohanan 2011, Pradeep \& Vrinda } \\
2011 \text { as Copelandia cyanescens (Berk. \& Broome) } \\
\text { Singer\}. }\end{array}$ \\
\hline Panaeolus fimicola (Pers.) Gillet & Kol & Pradeep \& Vrinda 2011. \\
\hline Panaeolus indicus Sathe \& J.T. Daniel^ ${ }^{\wedge}$ & Kot & Sathe \& Daniel 1979, Florence 2004. \\
\hline Panaeolus papilionaceus (Bull.) Quél. & Kol, Thi & $\begin{array}{l}\text { Pradeep \& Vrinda } 2011 \text { \{also as Panaeolus sphinctrinus } \\
\text { (Fr.) Quél.\}. }\end{array}$ \\
\hline Panaeolus rickenii Hora & Pal, Kol, Way & Pradeep \& Vrinda 2011. \\
\hline Panaeolus subbalteatus (Berk. \& Broome) Sacc. & $\mathrm{Pal}$ & Mohanan 2011. \\
\hline \multicolumn{3}{|l|}{ BOLETALES } \\
\hline \multicolumn{3}{|l|}{ GOMPHALES } \\
\hline \multicolumn{3}{|l|}{ Gomphaceae } \\
\hline Gloeocantharellus lateritius (Petch) Corner & Mal & Joseph \& Manimohan 1998. \\
\hline \multicolumn{3}{|l|}{ POLYPORALES } \\
\hline Lentinus bambusinus T.K.A. Kumar \& Manim.^^ & Mal & Kumar \& Manimohan 2005. \\
\hline Lentinus caespiticola Pat. \& Har. & Mal & $\begin{array}{l}\text { Manimohan \& Leelavathy } 1995 \text { \{as L. caespiticola var. } \\
\text { asiaticus Manim. Leelav. ^\}, Manimohan et al. } 2004 .\end{array}$ \\
\hline
\end{tabular}


Mycosphere Doi 10.5943/mycosphere/4/1/6

\begin{tabular}{|c|c|c|}
\hline Taxa & Distribution* & References \\
\hline${\text { Lentinus dicholamellatus Manim. }{ }^{\wedge}}$ & Mal, Thi, Way & $\begin{array}{l}\text { Manimohan et al. 2004, Pradeep \& Vrinda 2007, Varghese } \\
\text { et al. 2010, Mohanan 2011. }\end{array}$ \\
\hline Lentinus giganteus Berk. & Thi, Mal, Kol & $\begin{array}{l}\text { Joseph et al. 1995, Manimohan et al. 2004, Pradeep \& } \\
\text { Vrinda 2007, Mohanan } 2011 .\end{array}$ \\
\hline Lentinus patulus Lév. & Mal & Mohanan 2011. \\
\hline Lentinus polychrous Lév. & Ern, Thi, Ern & Manimohan et al. 2004, Mohanan 2011. \\
\hline Lentinus sajor-caju (Fr.) Fr. & Kot, Thi, Way, Kol, Thr & $\begin{array}{l}\text { Sathe \& Daniel 1980, Florence 2004, Manimohan et al. } \\
\text { 2004, Pradeep \& Vrinda 2007, Varghese et al. 2010, } \\
\text { Mohanan 2011. As Pleurotus sajor-caju (Fr.) Singer in } \\
\text { Geetha et al. 1989, Florence \& Balasundaran 2000, Ouseph } \\
\text { et al. } 2001 \text { and Florence } 2004 .\end{array}$ \\
\hline Lentinus squarrosulus Mont. & $\begin{array}{l}\text { Ern, Thr, Thi, Pal, Mal, } \\
\text { Way, Ala, Kol }\end{array}$ & $\begin{array}{l}\text { Sharma et al. 1985, Sankran et al. 1995, Florence \& } \\
\text { Yesodharan 2000, Florence 2004, Manimohan et al. 2004, } \\
\text { Pradeep \& Vrinda 2007, Varghese et al. 2010, Mohanan } \\
\text { 2011. }\end{array}$ \\
\hline Lentinus strigosus Fr. & Way, Kan, Mal & Manimohan et al. 2004, Mohanan 2011. \\
\hline Lentinus tigrinus (Bull.) Fr. & Ern, Way & Mohanan 2011. \\
\hline Lentinus tuber-regium $(F r) F$.$r .$ & Thi & $\begin{array}{l}\text { Geetha et al., 2002; Florence, } 2004 \text { (as Pleurotus tuber- } \\
\text { regium (Rumph. ex Fr.) Singer }\end{array}$ \\
\hline Panus hookerianus (Berk.) T.W. May \& A.E. Wood & Mal & Manimohan et al., 2004 (as Lentinus hookerianus Berk.). \\
\hline Panus indicus Sathe \& J.T. Daniel ${ }^{\wedge}$ & Kot & Sathe \& Daniel 1980, Florence 2004. \\
\hline Panus similis (Berk. \& Broome) T.W. May \& A.E. Wood & Way, Kan, Thi & $\begin{array}{l}\text { Manimohan et al. } 2004 \text { (as Lentinus similis Berk. \& } \\
\text { Broome). }\end{array}$ \\
\hline \multicolumn{3}{|l|}{ RUSSULALES } \\
\hline \multicolumn{3}{|l|}{ Auriscalpiaceae } \\
\hline Lentinellus pulvinulus (Berk.) Pegler & Mal & $\begin{array}{l}\text { Joseph \& Manimohan } 1998 \text { as Lentinellus pseudobarbatus } \\
\text { (Petch) Pegler\}. }\end{array}$ \\
\hline \multicolumn{3}{|r|}{ (1) } \\
\hline Lactarius ignifluus K.B. Vrinda \& C.K. Pradeep^^ & Ern,Way, Thi, Kol & $\begin{array}{l}\text { Vrinda et al. 2002b, Pradeep \& Vrinda 2007, Mohanan } \\
2011 .\end{array}$ \\
\hline Lactarius nebulosus Pegler & Way & Mohanan 2011. \\
\hline Lactarius uyedae Singer & Thi & Vrinda et al. 2000a. \\
\hline Russula aciculocystis Kauffman ex Bills \& O.K. Mill. & Thi & Pradeep \& Vrinda 2010, Mohanan 2011. \\
\hline Russula adusta (Pers) Fr. & Mal, Thi & Pradeep \& Vrinda 2010, Mohanan 2011. \\
\hline Russula amoena Quél. & Pal, Kol, Way, Thi & $\begin{array}{l}\text { Vrinda et al. 1997c, Pradeep \& Vrinda 2010, Mohanan } 2011 \\
\text { (as Russula mariae Peck). }\end{array}$ \\
\hline Russula atropurpurea (Krombh.) Britzelm. & Mal & Mohanan 2011. \\
\hline Russula cinerella Pat. & Way & Mohanan 2011. \\
\hline Russula congoana Pat. & Ern, Kol, Mal, Way, Thi & $\begin{array}{l}\text { Pradeep \& Vrinda 2010, Varghese et al. 2010, Mohanan } \\
2011 .\end{array}$ \\
\hline
\end{tabular}


Mycosphere Doi 10.5943/mycosphere/4/1/6

\begin{tabular}{|c|c|c|}
\hline Taxa & Distribution* & References \\
\hline Russula delicula Romagn. & Thi, Way & Pradeep \& Vrinda 2010, Mohanan 2011. \\
\hline Russula grata Britzelm. & Thi & Pradeep \& Vrinda 2010 (as Russula laurocerasi Melzer). \\
\hline Russula hygrophytica Pegler & Ern, Thi & Mohanan 2011. \\
\hline Russula indica Sathe \& J.T. Daniel^ ${ }^{\wedge}$ & Idu & Sathe \& Daniel 1980, Florence 2004. \\
\hline Russula leelavathyi K.B. Vrinda, C.K. Pradeep \& T.K. Abraham^^ & Thi, Mal, Way & $\begin{array}{l}\text { Vrinda et al. 1997a, Pradeep \& Vrinda 2007, Pradeep \& } \\
\text { Vrinda 2010, Mohanan } 2011 .\end{array}$ \\
\hline Russula luteotacta Rea & Thi, Ern & $\begin{array}{l}\text { Pradeep \& Vrinda 2010, Mohanan 2011, Pradeep \& Vrinda } \\
2011 .\end{array}$ \\
\hline Russula martinica Pegler & Mal & Mohanan 2011 \\
\hline Russula michiganensis Shaffer & Way, Ern & Mohanan 2011. \\
\hline Russula periglypta Berk. \& Broome & Thi, Mal & $\begin{array}{l}\text { Pradeep \& Vrinda 2010, Manimohan \& Deepna Latha 2011, } \\
\text { Mohanan } 2011 .\end{array}$ \\
\hline Russula purpureonigra Petch & Thi, Mal & Pradeep \& Vrinda 2010, Manimohan \& Deepna Latha 2011. \\
\hline Russula romagnesiana Schaffer & Thi & Pradeep \& Vrinda 2010. \\
\hline
\end{tabular}

* Districts of Kerala State (see Fig 1): Kas = Kasargod; Kan = Kannur; Way = Wayanad; Koz = Kozhikode; Mal = Malappuram; Thr = Trissur; Pal = Palakkad $;$ Ern = Ernakulam; Idu = Idukki; Kot = Kottayam; Ala = Alappuzha; Pat = Pathanamthitta; Kol $=$ Kollam; Thi $=$ Thiruvananthapuram.

@ Names not found in Index Fungorum.

$\wedge$ Taxa described as new to science from Kerala 


\section{Acknowledgements}

We are greatly indebted to Dr T.K. Arun Kumar for critically examining the manuscript and verifying the accuracy of several entries in this checklist. We are also thankful to Dr K.B. Vrinda and Dr C.K. Pradeep for bringing to our attention a few omissions in an earlier version of this manuscript. VA Farook acknowledges support from the Kerala State Council for Science, Technology and Environment in the form of a Research Fellowship. SS Khan acknowledges support in the form of a Maulana Azad National Fellowship by the Ministry of Minority Affairs, Government of India.

\section{References}

Abraham TK, Pradeep NS. 1995 - Utilization of a common weed Chromolaena odorata (L.) King \& Robinson as a substrate for oyster mushroom cultivation. Mushroom Research 4, 8184.

Abraham TK, Vrinda KB, Pradeep CK, Joseph AV. 1995 - Edible cantherelle from South India. Mushroom Research 4, 73-76.

Abraham TK, Vrinda KB, Pradeep CK. 1996 A new species of Hygrocybe from India. Mycotaxon 60, 361-364.

Aravindakshan DM, Manimohan P. 2010 Amparoina spinosissima: a continental Asian record and some taxonomic observations. Mycotaxon 114, 49-54.

Arnolds EJM, Leelavathy KM, Manimohan P. 2004 - Pseudobaespora lavendulamellata, a new species from Kerala, India. Persoonia 18(3), 435438.

Balakrishnan B, Nair MC. 1998 - Tricholoma lobayense Heim, a new edible mushroom to Kerala. In: Frontiers in Mushroom Research (eds TK Abraham, NS Pradeep, P Pushpangadan). Tropical Botanical Garden and Research Institute,

Thiruvananthapuram, Kerala, 75-78.

Bhavanidevi S. 1998 - Agaricales of Kerala. In: Frontiers in Mushroom Research (eds TK Abraham, NS Pradeep, P Pushpangadan). Tropical Botanical
Garden and Research Institute, Thiruvananthapuram, Kerala, 63-74.

Bhavanidevi S, Nair MC. 1983 - Addition to Indian Agaricales. In: Indian Mushroom Science-II (eds TN Kaul, BM Kapoor). CSIR, New Delhi, 271274.

Bilgrami KS, Jamaluddin S, Rizwi MA. 1991 Fungi of India. List and References. Today and Tomorrow's Printers and Publishers, New Delhi.

Chandramohanan R, Kaveriappa KM. 1983 White thread blight of cacao in India. Indian Phytopathology 36, 394-396.

Deepa S, Vrinda KB, Pradeep CK. 2006a Additions to leucocoprinoid fungi from Western Ghats of Kerala. Mushroom Research 15(1), 1-5.

Deepa S, Vrinda KB, Pradeep CK. 2006b More leucocoprinoid fungi from the Western Ghats. Mushroom Research 15(2), 103-110.

Deepa S, Vrinda KB, Pradeep CK. 2009 Additions to the genus Leucoagaricus from Kerala state, India. Journal of Mycopathological Research 47(2), 119-128.

Florence EJM, Biodiversity Documentation for Kerala, Part 2: Microorganisms (Fungi). KFRI Handbook No.17. Kerala Forest Research Institute, Peechi, Kerala, India.

Florence EJM, Balasundaran M. 2000 Mushroom cultivation using forest litter and waste wood. KFRI Research Report No. 195. Kerala Forest Research Institute, Peechi, Kerala, India.

Florence EJM, Yesodharan K. 1997 - Status survey of macrofungal flora of PeechiVazhani wildlife sanctuary. In: Proceedings of Ninth Kerala Science Congress (ed PK Iyengar). STEC, Thiruvananthauram, 477-479.

Florence EJM, Yesodharan K. 2000 Macrofungal flora of Peechi-Vazhani wildlife sanctuary. KFRI Research Report No. 191. Kerala Forest Research Institute, Peechi, Kerala, India.

Geetha D, Sivaprakasam K. 1998 - Enzyme and sporophore production potential of oyster mushroom (Pleurotus spp.). Mushroom Research 7(1), 39-42. 
Geetha D, Nair MC, Bhavanidevi S. 1989 Mushroom mycelia as poultry feed. Indian Phytopathology 42(3), 462-463.

Geetha D, Sherin AS, Gokulapalan C, Suhrban M. 2002 - Pleurotus tuber-regium - a new promising mushroom for the tropics. Mushroom Research 11(2), 7376.

Heinemann P, Leelavathy KM. 1991 - The genus Micropsalliota (Agaricaceae) in Kerala State, India, Mycological Research 95(3), 341-346.

Heinemann P, Little Flower 1983 Micropsalliota de Kerala (Inde). Bulletin du Jardin Botanique National de Belgique 53, 75-84.

Heinemann P, Little Flower. 1984 Hymenagaricus (Agaricaceae) de Kerala (Inde) et de Sri Lanka. Bulletin $\mathrm{du}$ Jardin Botanique National de Belgique 54 (1/2), 151-182.

Joseph AV, Manimohan P. 1998 - Rediscovery of two rare agaricoid basidiomycetes. Mycological Research 102(4), 476478.

Joseph AV, Abraham TK, Vrinda KB, Pradeep CK. 1995 - New agarics from South India. Mushroom Research 4, 1-6.

Justin PSJ, Abraham TK. 1998 - Utilization of some common weeds for the cultivation of mushrooms. Mushoom Research 7(2), 99-100.

Kumar TKA, Manimohan P. 2004 - A new species of Leucocoprinus from India. Mycotaxon 90(2), 393-397.

Kumar TKA, Manimohan P. 2005 - A new species of Lentinus from India. Mycotaxon 92, 119-123.

Kumar TKA, Manimohan P. 2009a - The genus Lepiota (Agaricales, Basidiomycota) in Kerala State, India. Mycotaxon 107, 105-138.

Kumar TKA, Manimohan P. 2009b - The genus Cystolepiota (Agaricales, Basidiomycota) in Kerala State, India. Mycotaxon 107, 277-284.

Kumar TKA, Manimohan P. 2009c - The genera Leucoagaricus and Leucocoprinus (Agaricales, Basidiomycota) in Kerala State, India. Mycotaxon 108, 385-428.
Kumar TKA, Manimohan P. 2009d Rediscovery of Trogia cyanea and a record of $T$. infundibuliformis (Marasmiaceae, Agaricales) from Kerala State, India. Mycotaxon 109, 429-436.

Lakshmanan L, Bhavanidevi S, Nair MC. 1979 - Occurrence of Thanatephorus cucumeris - as a weed fungus on mushroom beds. Current Science 48(12), 543-544.

Leelavathy KM, Little Flower. 1981 Additions to South Indian Agaricales-I. Kavaka 9, 31-33.

Leelavathy KM, Little Flower. 1986 - The genus Micropsalliota in India. Kavaka 14, 17-23.

Leelavathy KM, Little Flower, Suja CP. 1983 The genus Termitomyces in India. In: Indian Mushroom Science-II (eds TN Kaul, BM. Kapoor). CSIR, New Delhi, 402-407.

Leelavathy KM, Manimohan P, Arnolds EJM. 2006 - Hygrocybe in Kerala State, India. Persoonia 19(1), 101-151.

Leelavathy KM, Zachariah S, Sankaran KV. 1981 - Clarkeinda trachodes - an agaric new to India. Mycologia 73(1), 204-207.

Little Flower, Hosagoudar VB, Abraham TK. 1997 - Xanthagaricus, a new generic name in the family Agaricaceae. New Botanist 24, 93-100.

Manimohan P, Arnolds E. 1998 - Dermoloma cystidiatum, a new species of Dermoloma (Agaricales) from India. Persoonia 17(1), 149-152.

Manimohan P, Deepna Latha KP. 2011 Observations on two rarely collected species of Russula. Mycotaxon 116, 125-131.

Manimohan P, Leelavathy KM. 1987a Marasmius parvulus sp. nov. (Tricholomataceae) from South India. Transactions of the British Mycological Society 88(3), 422-423.

Manimohan P, Leelavathy KM. 1987b Marasmius flexipes sp. nov. (Tricholomataceae) from South India. Transactions of the British Mycological Society 89(2), 264-265. 
Manimohan P, Leelavathy KM. 1987c Pleuromycenula hydrophila sp. nov. (Tricholomataceae) from South India. Transactions of the British Mycological Society 89(1), 116-117.

Manimohan P, Leelavathy KM. 1988a - New agaric taxa from Southern India. Transactions of the British Mycological Society 91(4), 573-576.

Manimohan P, Leelavathy KM. 1988b - Two new species of Alboleptonia (Agaricales, Entolomataceae) from Southern India. Transactions of the British Mycological Society 91(4), 710-712.

Manimohan P, Leelavathy KM. 1988c Mycena indica, a new species from Southern India. Mycologia 80(6), 861862.

Manimohan P, Leelavathy KM. 1988d Mniopetalum minutum sp. nov. (Tricholomataceae) from South India. Transactions of the British Mycological Society 90(1), 142.

Manimohan P, Leelavathy KM. 1988e Marasmiellus fusicystis sp. nov. (Tricholomataceae) from South India. Transactions of the British Mycological Society 90(1), 143-144.

Manimohan P, Leelavathy KM. 1989a Marasmius species new to India. Sydowia 41, 185-199.

Manimohan P, Leelavathy KM. 1989b - Some Agarics new to India. Sydowia 41, 200-208.

Manimohan P, Leelavathy KM. 1989c - Two new varieties of Mycena alphitophora from Southern India. Mycological Research 93(1), 118-120.

Manimohan P, Leelavathy KM. 1989d - New records of Inocybe from India. Calicut University Reserarch Journal 1(2), 1-3.

Manimohan P, Leelavathy KM. 1995 - A new variety of Lentinus caespiticola from Southern India. Mycological Research 99(4), 451-452.

Manimohan P, Thomas KA. 1998 - A new species of Gloiocephala from India. Mycotaxon. 69, 87-91.

Manimohan P, Vrinda KB, Leelavathy KM. 1988 - Rare agarics from Southern India. Kavaka 16 (1, 2), 50-56.
Manimohan P, Joseph AV, Leelavathy KM. 1995 - The genus Entoloma in Kerala State, India. Mycological Research 99(9), 1083-1097.

Manimohan P, Leelavathy KM, Noordeloos ME. 2002 - Three new species of Entoloma from Kerala State, India. Persoonia 17(4), 625-630.

Manimohan P, Divya N, Kumar TKA, Vrinda KB, Pradeep CK - 2004. The genus Lentinus in Kerala State, India. Mycotaxon 90(2), 311-318.

Manimohan P, Noordeloos M.E, Dhanya AM. 2006 - Studies on the genus Entoloma (Basidiomycetes, Agaricales) in Kerala State, India. Persoonia 19(1), 45-93.

Manimohan P, Thomas KA, Nisha VS. 2007 Agarics on elephant dung in Kerala State, India. Mycotaxon 99, 147-157.

Manimohan P, Kumar TKA, Vrinda KB, Pradeep CK. 2010 - Megacollybia virosa, a new species with toxic basidiomata from India. Mycotaxon 111, 363-368.

Mešić A, Tkalčec Z. 2010 - New names in the genus Marasmius. Mycotaxon 113, 283-285.

Mohanan C. 2003 - Mycorrhizae in forest plantations: association, diversity and exploitation in planting improvement. KFRI Research Report No. 252. Kerala Forest Research Institute, Peechi, Kerala, India.

Mohanan C. 2011 - Macrofungi of Kerala. KFRI Handbook No. 27, Kerala Forest Research Institute, Peechi, Kerala, India.

Nag Raj TR. 1965 - Thread blight of water hyacinth. Current Science 34(21), 618619.

Natarajan K, Manjula B. 1982 - South Indian Agaricales - XVIII - Marasmius. Kavaka 10, 13-28.

Natarajan K, Raman N. 1983 - South Indian Agaricales. J. Cramer, Vaduz, Germany.

Noordeloos ME, Vrinda KB, Manimohan P. 2007 - On two remarkable brownspored agarics from Kerala State, India. Fungal Diversity 27, 145-155.

Ouseph A, Geetha D, Suharban M. 2001 Lignocellulose degradation by oyster 
mushroom. Mushroom Research 10(1), 37-40.

Peintner U, Moser MM, Thomas KA, Manimohan P. 2003 - First records of ectomycorrhizal Cortinarius species (Agaricales, Basidiomycetes) from tropical India and their phylogenetic position based on rDNA ITS sequences. Mycological Research 107 (4), 485494.

Pradeep CK, Vrinda KB. 2005a - New records of Pluteus Fr. from Kerala. Mushroom Research. 14(1), 1-4.

Pradeep CK, Vrinda KB. 2005b - New additions to the genus Pluteus Fr. from India. Mushroom Research 14(2), 4649.

Pradeep CK, Vrinda KB. 2006 - New and noteworthy species of Pluteus (Pluteaceae, Agaricales) from Kerala State, India. Persoonia 19(1), 95-99.

Pradeep CK, Vrinda KB. 2007 - Some noteworthy agarics from Western Ghats of Kerala. Journal of Mycopathological Research (1), 1-14.

Pradeep CK, Vrinda KB. 2008 - Two new Pluteus (Basidiomycotina, Agaricales) from Kerala, India. Nova Hedwigia 87(1-2), 231-236.

Pradeep C.K, Joseph AV, Abraham TK, Vrinda KB - 1996a. New records of Agaricales from India. Journal of Economic and Taxonomic Botany. 20(1), 233-239.

Pradeep C.K, Joseph AV, Abraham TK, Vrinda KB - 1996b. Hygrocybe punicea - an edible mushroom new to India. Acta Botanica Indica 24, 103104.

Pradeep CK, Vrinda KB, Mathew S, Abraham TK. 1998 - The genus Volvariella in Kerala State, India. Mushroom Research 7(2), 53-62.

Pradeep CK, Vrinda KB, Abraham TK. 2002 Pluteus section Pluteus (Pluteaceae, Agaricales) from Kerala State, India. Mycotaxon 83, 59-66.

Prdeep CK, Vrinda KB, Varghese SP. 2008 Favolaschia - a new genus record for India. Mushroom Research 17(2), 6768.

Reddy CS, Pattanaik C, Reddy KN, Raju VS.
2007 - Census of endemic flowering plants of Kerala, India. Journal of Plant Sciences, 2, 489-503.

Sankaran KV. 1993 - Collybia leucophaea an addition to Indian agaric flora. Acta Botanica Indica. 25, 127-128.

Sankaran KV, Florence EJM. 1995 Macrofungal flora and checklist of plant diseases of Malayattoor forests (Kerala). Advances in Forestry Research in India. Vol. 12 (ed SK Mukherjee) International Book Distributors, Dehra Dun, 147-168.

Sathe AV, Daniel J. 1980 - Agaricales (Mushrooms) of Kerala State. MACS Monograph. No. 1, 75-108.

Sharma JK, Mohanan C, Florence EJM. 1985 Disease survey in nurseries and plantations of forest tree species grown in Kerala. KFRI Research Report No. 36. Kerala Forest Research Institute, Peechi, Kerala, India.

Thomas KA, Manimohan P. 2001 - A new species of Pholiota from India. Mycotaxon 78, 181-184.

Thomas KA, Manimohan P. 2003 - The genus Agrocybe in Kerala State, India. Mycotaxon 86, 317-333.

Thomas KA, Hausknecht A, Manimohan P 2001. Bolbitiaceae of Kerala State, India: New species and new and noteworthy records. Österreichische Zeitschrift für Pilzkunde 10, 87-114.

Thomas KA, Manimohan P, Guzmán G, Tapia F, Ramirez-Guillen F. 2002a - The genus Psilocybe in Kerala State, India. Mycotaxon 83, 195-207.

Thomas KA, Peintner U, Moser MM, Manimohan P. 2002b - Anamika, a new mycorrhizal genus of Cortinariaceae from India and its phylogenetic position based on ITS and LSU sequences. Mycological Research 106 (2), 245251.

Thomas KA, Guzman-Davalos L, Manimohan P. 2003 - A new species and new records of Gymnopilus from India. Mycotaxon 85, 297-305.

Varghese SP, Pradeep CK, Vrida KB. 2010 Mushrooms of tribal importance in Wayanad area of Kerala. Journal of Mycopathological Research 48(2), 
311-320.

Vrinda KB, Leelavathy KM. 1996 - New records of Agaricus from India. Journal of Economic and Taxonomic Botany 20(2), 345-349.

Vrinda KB, Pradeep CK. 2006 - Macrocybe lobayensis, an edible mushroom from Western Ghats of Kerala. Mushroom Research 15(2), 157-158.

Vrinda KB, Pradeep CK. 2009 - Termitomyces sagittiformis - a lesser known edible mushroom from the Western Ghats. Mushroom Research 18(1), 33-36.

Vrinda KB, Pradeep CK. 2010 Ectomycorrhizal fungal diversity in three different forest types and their association with endemic, indigenous and exotic species in the Western Ghat forests of Thiruvananthapuram district, Kerala. Journal of Mycopathological Research 48(2), 279- 289.

Vrinda KB, Pradeep CK. 2011 - Toxic and hallucinogenic mushrooms of Kerala. Journal of Mycopathological Research 49(2), 231-246.

Vrinda KB, Pradeep CK, Pradeep NS, Abraham TK. 1995 - New records of Hygrocybe from India. Journal of Economic and Taxonomic Botany. 19(3), 576-584.

Vrinda KB, Pradeep CK, Abraham TK. 1996a - Agaricales from Western Ghats-III. Mushroom Research 5, 47-50.

Vrinda KB, Pradeep CK, Joseph AV, Abraham TK. $1996 \mathrm{~b}-\mathrm{A}$ new Inocybe (Cortinariaceae) from Kerala State, India. Mycotax on 57, 171-174.

Vrinda KB, Pradeep CK, Pratheep NS, Abraham TK. 1996c - Agaricales from Western Ghats - I. Journal of Mycopathological Research 34(2), 119-123.

Vrinda KB, Pradeep CK, Abraham TK. 1997a - A new species of Russula from Kerala, India. Mycotaxon 62, 389-393.

Vrinda KB, Pradeep CK, Abraham TK. 1997b - Some Inocybes new to India. Journal of Economic and Taxonomic Botany 21(1), 41-45.

Vrinda KB, Pradeep CK, Mathew S, Abraham TK. 1997c - Agaricales from Western Ghats-IV. Journal of Mycology and
Plant Pathology 27(3), 346-350.

Vrinda KB, Pradeep CK, Mathew S, Abraham TK. 1997d - Agaricales from Western Ghats-V. Mushroom Research 6(1), 710.

Vrinda KB, Pradeep CK, Mathew S, Abraham TK. $1997 \mathrm{e}$ - Inocybe purpureoflavida sp. nov. (Cortinariaceae) from Western Ghats of Kerala State, India. Mycotax on 64, 1-6.

Vrinda KB, Pradeep CK, Pradeep NS, Abraham TK. 1997f - Agaricales from Western Ghats-II. Indian Journal of Forestry 20(4), 314-318.

Vrinda KB, Pradeep CK, Abraham TK. 1999a - Bioluminescent agarics from Western Ghats. Mushroom Research 8(2), 3133.

Vrinda KB, Pradeep CK, Mathew S, Abraham TK. 1999b - Agaricales from Western Ghats-VI. Indian Phytopathology 52(2), 198-200.

Vrinda KB, Pradeep CK, Mathew S, Abraham TK. 1999c - Agaricales from Western Ghats-VII. Mushroom Research 8(2), 9-12.

Vrinda KB, Pradeep CK, Mathew S, Abraham TK. 2000a - Some pleurotoid agarics from Western Ghats, Kerala. Journal of Mycopathological Research 38(1), 4547.

Vrinda KB, Pradeep CK, Mathew S, Abraham TK. 2000b - Agaricales from Western Ghats-VIII. Journal of Mycopathological Research 38(2), 97-100.

Vrinda KB, Pradeep CK, Mathew S, Abraham TK. 2000c - Catatrama (Tricholomataceae), a genus new to India. Persoonia 17(3), 495-496.

Vrinda KB, Pradeep CK, Abraham TK. 2001 Additions to Indian mushroom flora. Mushroom Research 10(1), 1-4.

Vrinda KB, Pradeep CK, Abraham TK. 2002a - Termitomyces umkowaani (Cooke \& Mass.) Reid - an edible mushroom from the Western Ghats. Mushroom Research 11(1), 7-8.

Vrinda KB, Pradeep CK, Mathew S, Abraham TK. 2002b - Lactarius ignifluus (Russulaceae), a new species from India. Persoonia 18(1), 129-133.

Vrinda KB, Pradeep CK, Abraham TK. 2003a 
- Psathyrella velutina (Pers. ex Fr.) Sing. - an edible mushroom from the Western Ghats. Mushroom Research 12(1), 17-18.

Vrinda KB, Pradeep CK, Deepa S, Abraham TK. 2003b. Some leucocoprinoid fungi from the Western Ghats. Mushroom Research 12(1), 1-7.

Vrinda KB, Pradeep CK, Kumar SS. 2005a Occurrence of a lesser known edible Amanita in the Western Ghats of Kerala. Mushroom Research 14(1), 58.

Vrinda KB, Prdeep CK, Kumar SS. 2005b Occurrence of the deadly Amanita phalloides in the Western Ghats of Kerala. Journal of Mycopathological Research 43(2), 259-262.

Vrinda KB, Pradeep CK, Varghese SP. 2009 Noteworthy records of Hygrocybe section Firmae from Western Ghats of Kerala. Mushroom Research 18(1), 14.

Watling R, Little Flower, Leelavathy KM. 1988 - Observations on the Bolbitiaceae - 27. Bolbitius and Conocybe in India with particular reference to the State of Kerala. In: Advances in Mycology (ed. GS Rawla). Chandigarh, India, 28-43. 